\title{
Relative Topological Entropy for Actions of Non-discrete Groups on Compact Spaces in the Context of Cut and Project Schemes
}

\section{T. Hauser ${ }^{1}$}

Received: 5 October 2019 / Revised: 21 February 2020 / Published online: 25 March 2020

(c) The Author(s) 2020

\begin{abstract}
In the study of aperiodic order via dynamical methods, topological entropy is an important concept. In this paper, parts of the theory, like Bowen's formula for fibre wise entropy or the independence of the definition from the choice of a Van Hove sequence, are extended to actions of several non-discrete groups. To establish these results, we will show that the Ornstein-Weiss lemma is valid for all considered groups which appear in the study of cut and project schemes.
\end{abstract}

Keywords Entropy · Ornstein-Weiss lemma - Bowen formula $\cdot$ Uniform space $\cdot$ Dynamical system $\cdot$ Cut and project scheme $\cdot$ Amenable group $\cdot$ Van Hove sequence $\cdot$ Følner sequence $\cdot$ Uniform lattice

Mathematics Subject Classification 37B40 $\cdot 37 \mathrm{~A} 35 \cdot 52 \mathrm{C} 23$

\section{Introduction}

Aperiodic order, an intermediate concept between order and disorder, has attracted a lot of attention over the last three decades in the fields of physics, geometry, number theory and harmonic analysis $[1,2,4,7-9,44,49]$. The construction of aperiodic point sets via cut and project schemes was pioneered by Yves Meyer in his famous monograph on "Algebraic numbers and harmonic analysis". For details see [39, Chapter II.5]. A cut and project scheme $(C P S)$ is a triple $(G, H, \Lambda)$, where $G$ and $H$ are locally compact amenable groups, ${ }^{1} \Lambda$ is a

\footnotetext{
1 A topological group $G$ is called locally compact whenever every neighbourhood of some element contains a compact neighbourhood of this element. For the notion of amenability see Sect. 2.4.

T. Hauser

till.hauser@uni-jena.de

1 Institute of Mathematics, Faculty of Mathematics and Computer Science, Friedrich Schiller University Jena, 07743 Jena, Germany
} 
uniform lattice ${ }^{2}$ in $G \times H$ and the projections $\pi_{G}$ and $\pi_{H}$ satisfy the following properties. The restriction $\left.\pi_{G}\right|_{\Lambda}$ is injective and $\pi_{H}(\Lambda)$ is dense in $H$. Then $G$ is called the physical space and $H$ is referred to as the internal space of $(G, H, \Lambda)$. Given a relatively compact subset $W \subseteq H$ with nonempty interior, usually called a window in this context, such a CPS produces a subset of $G$ via $\omega:=\pi_{G}(\Lambda \cap(G \times W))$. Subsets of $G$ that arise by this construction are called model sets. These sets are aperiodic, but have a longe range order, due to their algebraic origin. For further details and references on these notions see [3, Chapter 7]. CPS are usually studied under the assumption of commutativity. Nevertheless recent interest in the non commutative case, for example in [6] motivated us to omit the assumption of commutativity in our definition of CPS.

Model sets can be studied by methods of dynamical systems. One first introduces a compact Hausdorff topology on the set of all closed subsets of $G$ and shows that the set of all translations $\{\omega g ; g \in G\}$ is a pre-compact subset. Denote by $X$ the closure of $\{\omega g ; g \in G\}$. One then shows that $G \times X \ni(g, M) \mapsto M g:=\{m g ; m \in M\}$ is a dynamical system, referred to as the Delone dynamical system of $\omega$. For details on this construction see [7] in combination with [3]. In the study of model sets it is natural to study the topological entropy of this dynamical system as a measure of "complexity" of $\omega[4,9]$.

It is thus natural to ask for the validity of analogues of statements from the theory of entropy of $\mathbb{Z}$-actions. In $[33,43,58,60,61]$ such analogues are proven for actions of countable discrete amenable groups $G$. Nevertheless in the context of Delone dynamical systems $G$ is typically not discrete and we will see in Example 3.4 that there can be choices of $G$ that do not contain uniform lattices. In fact the absence of the possibility to restrict to discrete subgroups was one of the motivations of Meyer to construct CPS [39, Chapter II]. The references known to us for a systematic treatment of entropy theory of actions of non discrete groups are $[51,55]$. Both notions are not equivalent for actions of $\mathbb{R}^{d}$ with $d \geq 2$ and we will focus on the notion of Tagi-Zade, which is used in the context of aperiodic order [9]. Tagi-Zade presents entropy theory of $\mathbb{R}^{d}$ actions, but parts like fibre wise entropy, relative topological entropy, the freedom in the averaging in the definition of entropy are not addressed. Furthermore Bowen's formula, which states that the topological entropy of an action is less than the sum of the topological entropy of a factor and the relative topological entropy of the factor map [10], are not considered. These parts of the theory are addressed in the study of aperiodic order in $[9,32,34]$ and the importance of Bowen's formula for $\mathbb{R}^{d}$ actions comes up in [23, Remark 2.9.] and [23, Lemma 4.1.(ii)].

In order to define topological entropy of discrete amenable groups one uses a technique referred to as "Ornstein-Weiss lemma" $[37,43,57,58]$. In order to explain this in more detail we need the following notions. Let $G$ be an amenable group $G$. Denote by $\mathcal{K}(G)$ the set of all compact subsets of $G$. A function $f: \mathcal{K}(G) \rightarrow \mathbb{R}$ is called subadditive, if for all disjoint $A, B \in \mathcal{K}(G)$ there holds $f(A \cup B) \leq f(A)+f(B)$. Furthermore $f$ is said to be right invariant, if for all $A \in \mathcal{K}(G)$ and for all $g \in G$ there holds $f(A g)=f(A)$. A function $f$ is called monotone, if for all $A, B \in \mathcal{K}(G)$ with $A \subseteq B$ there holds $f(A) \leq f(B)$. $G$ is said to satisfy the Ornstein-Weiss lemma, if for any subadditive, right invariant and monotone function $f: \mathcal{K}(G) \rightarrow \mathbb{R}$ the limit

$$
\lim _{i \in I} \frac{f\left(A_{i}\right)}{\mu\left(A_{i}\right)}
$$

exists, is finite and does not depend on the choice of the Van Hove net $\left(A_{i}\right)_{i \in I}$ in $G$.

${ }^{2}$ A discrete subgroup $\Lambda$ of a locally compact group $G$ is called a uniform lattice, whenever it is co-compact, i.e. whenever $G / \Lambda$ is compact. 
The ideas behind this technique go back to [29,33,37,43,57]. In M. Gromovs extremly influential work [29] a sketch of a proof is presented for very general groups, but a rigorous proof is given only in the context of discrete amenable groups. For details see $[13,36,58]$ and Remark 3.1(ii). We thus give a rigorous proof of the Ornstein-Weiss lemma for groups arising in our context. The next theorem shows that all compactly generated abelian groups satisfy the Ornstein-Weiss lemma.

Theorem 1.1 Every amenable group containing a uniform lattice satisfies the Ornstein-Weiss lemma.

If $(G, H, \Lambda)$ is a CPS we know that $G \times H$ contains a uniform lattice and in order to show that $G$ satisfies the Ornstein-Weiss lemma one could hope that the properties of a CPS also imply that $G$ contains a uniform lattice. However in Example 3.4, which is a special case of examples, studied by Meyer in [39, Chapter II.10], we present a CPS with a physical space that does not contain any uniform lattice. Nevertheless we obtain that physical spaces of CPS satisfy the Ornstein-Weiss lemma from the next result.

Theorem 1.2 If $G$ and $H$ are amenable groups such that $G \times H$ satisfies the Ornstein-Weiss lemma, then $G$ and $H$ satisfy the Ornstein-Weiss lemma.

Corollary 1.3 Let $G$ be a locally compact amenable group. If there is a CPS, such that $G$ is the respective physical space, then $G$ satisfies the Ornstein-Weiss lemma.

Naturally the question arises how restrictive the existence of such a CPS is. In fact in the commutative case it is shown by Y. Meyer, that $G$ is the physical space of a CPS, iff it is the physical space of a CPS with an euclidean internal space [39, Chapter II.5.10], and this holds iff there exists a Meyer set in $G$ [39, Chapter II.14]. A Meyer set ${ }^{3}$ is a discrete subset $\omega \subseteq G$ such that there are a finite set $F \subseteq G$ and a compact set $K \subseteq G$ that satisfy $K \omega=G$ and $\omega \omega^{-1} \subseteq F \omega$. Examples of Meyer sets are all model sets.

A Meyer set $\omega$ that is symmetric $\left(\omega=\omega^{-1}\right)$ and that contains the neutral element is called an approximate uniform lattice and the arguments from [39] also show that a locally compact abelian group $G$ is a physical space of a CPS iff $G$ contains a uniform approximate lattice [5]. This equivalence remains valid also in the context of connected nilpotent Lie groups [38], but the existence of a CPS under the assumption of the existence of a Meyer set seems open for general locally compact groups. An example of a metrizable and separable locally compact abelian group $G$ that contains no Meyer set and therefore is not a physical space of a CPS is given in [39, Chapter II.11].

Nevertheless we are interested in groups $G$ that contain Meyer sets and for those we can now define (relative) topological entropy for some action $\varphi$ of $G$ on a compact metric space $(X, d)$. For a compact subset $A \subseteq G$ define the Bowen metric for $x, y \in X$ as follows

$$
d_{A}(x, y):=\max _{g \in A} d(\alpha(g, x), \alpha(g, y)) .
$$

Furthermore for $M \subseteq X$ and $\varepsilon>0$ we denote the minimum cardinality of an open cover of $M$ consisting of sets of $d_{A}$-diameter ${ }^{4}$ strictly less than $\varepsilon$ by $\operatorname{cov}_{M}\left[d_{A}<\varepsilon\right]$. One then shows that $\mathcal{K}(G) \ni A \mapsto \log \left(\operatorname{cov}_{X}\left[d_{A}<\varepsilon\right]\right)$ is a monotone, right invariant and subadditive mapping and thus the Ornstein Weiss lemma can be applied to yield the existence of the

\footnotetext{
3 Note that these sets are the "harmonious and relatively dense" sets in [39].

4 The $d$-diameter of a set $M \subseteq X$ is defined by $\sup _{(x, y) \in M^{2}} d(x, y)$.
} 
following limit, as well as the independence from the choice of a Van Hove net. For some Van Hove net $\left(A_{i}\right)_{i \in I}$ one defines the topological entropy of $\varphi$ as

$$
\mathrm{E}(\varphi):=\sup _{\varepsilon>0} \lim _{i \in I} \frac{\log \left(\operatorname{cov}_{X}\left[d_{A_{i}}<\varepsilon\right]\right)}{\mu\left(A_{i}\right)} .
$$

Furthermore if $p: X \rightarrow Y$ is a factor map onto some action $\psi: G \times Y \rightarrow Y$ one defines with a similar argument the relative topological entropy (of $p$ ) as

$$
\mathrm{E}(\varphi \stackrel{p}{\rightarrow} \psi):=\sup _{\varepsilon>0} \lim _{i \in I} \frac{\log \left(\sup _{y \in Y} \operatorname{cov}_{p^{-1}(y)}\left[d_{A_{i}}<\varepsilon\right]\right)}{\mu\left(A_{i}\right)} .
$$

In particular there holds $\mathrm{E}(\varphi \stackrel{p}{\rightarrow} \psi)=\mathrm{E}(\varphi)$, whenever $Y$ is a single point.

It is standard to define the topological entropy of an action of $\mathbb{R}$ as the restriction to the action of $\mathbb{Z}$. We present next that in a similar way one obtains the relative topological entropy of an action as the scaled entropy of the restricted action to certain model sets and in particular any uniform lattice. This allows to transfer several Theorems proven for discrete amenable groups to our context.

To formulate the exact statement we need the following notions. We denote by $|F|$ the cardinality of a set $F$ and by $\mu$ the Haar measure on $G$. Furthermore we say, that a discrete subset $\Lambda \subseteq G$ has a well defined uniform density, if $\operatorname{dens}(\Lambda):=\lim _{i \in I} \frac{\left|\Lambda \cap A_{i}\right|}{\mu\left(A_{i}\right)}$ exists, is finite and is independent from the choice of the Van Hove net $\left(A_{i}\right)_{i \in I}$. We refer to dens $(\Lambda)$ as the uniform density of $\Lambda$. Note that every uniform lattice $\Lambda$ has a well defined uniform density, which is given by dens $(\Lambda):=\mu(C)^{-1}$, where $C$ denotes a fundamental domain of $\Lambda$. See Sect. 2 for details on this notion. Furthermore in locally compact abelian groups all regular ${ }^{5}$ model sets have a well defined uniform density [52, Corollary 15.1]. A subset $\Lambda \subseteq G$ is called relatively dense, if there holds $K \Lambda=G$ for some compact subset $K \subseteq G$. Note that all model sets are relatively dense. Denote for any map $f: A \rightarrow B$ and any subset $M \subseteq A$ by $\left.f\right|_{M}$ the restriction $\left.f\right|_{M}: M \rightarrow B: a \mapsto f(a)$. The following statement is contained in the statement of Theorem 5.2 in Sect. 5.

Theorem 1.4 Let $\varphi$ be an action of $G$ on a compact metric space $X$. Let furthermore $\psi$ be a factor of $\varphi$ via factor map $p: X \rightarrow Y$. Let $\Lambda$ be a relatively dense subset of $G$ and let $\left(A_{i}\right)_{i \in I}$ be a Van Hove net. Set $F_{i}:=A_{i} \cap \Lambda$.

(i) If $\Lambda$ has a well defined uniform density dens $(\Lambda)$, then there holds

$$
\mathrm{E}(\varphi \stackrel{p}{\rightarrow} \psi)=\operatorname{dens}(\Lambda) \sup _{\varepsilon>0} \limsup _{i \in I} \frac{\log \left(\sup _{y \in Y} \operatorname{cov}_{p^{-1}(y)}\left[d_{F_{i}}<\varepsilon\right]\right)}{\left|F_{i}\right|}
$$

(ii) If $\Lambda$ is a uniform lattice, then there holds

$$
\mathrm{E}(\varphi \stackrel{p}{\rightarrow} \psi)=\operatorname{dens}(\Lambda) \mathrm{E}\left(\left.\left.\varphi\right|_{\Lambda \times X} \stackrel{p}{\rightarrow} \psi\right|_{\Lambda \times Y}\right)
$$

We extend Bowens formula beyond $\mathbb{Z}$ actions in the next result to actions of compactly generated locally compact abelian groups such as $\mathbb{R}^{d}$ and $\mathbb{Z}^{d}$, which are the most common choices in the study of cut and project schemes.

5 A model set is said to be regular, if the Haar measure of the topological boundary of the corresponding window is 0 . 
Theorem 1.5 Let $\varphi, \psi$ and $\rho$ be actions of an amenable group containing a countable uniform lattice on compact Hausdorff spaces $X, Y$ and $Z$ respectively. Let $\psi$ be a factor of $\varphi$ via factor map $p$ and $\rho$ be a factor of $\psi$ via factor map $q$. Then there holds

$$
\max \{\mathrm{E}(\varphi \stackrel{p}{\rightarrow} \psi), \mathrm{E}(\psi \stackrel{q}{\rightarrow} \rho)\} \leq \mathrm{E}(\varphi \stackrel{q \circ p}{\rightarrow} \rho) \leq \mathrm{E}(\varphi \stackrel{p}{\rightarrow} \psi)+\mathrm{E}(\psi \stackrel{q}{\rightarrow} \rho) .
$$

The article is structured as follows. In Sect. 2 we fix some notion. Section 3 is devoted to the proof of the Theorems 1.1 and 1.2. In Sect. 4 we present basic results from the theory of relative topological entropy. As the mentioned topology in the construction of Delone dynamical systems is naturally defined via the notion of a uniformity $[7,50]$ and the corresponding arguments are well known [33,41,43,56-58,60,61], we took the freedom to follow an idea from $[19,30,41,59]$ and use the language of uniformities in order to prove the results for actions on compact Hausdorff spaces. The approach via uniformities also yields tools to provide the equivalence of the definitions given in [55], our and the classical definitions. In particular we show that the implicit dependencies on Van Hove nets in $[9,32,34]$ can be dropped. Theorem 1.4 is proven in Sect. 5. In Sect. 6 we present a proof of Theorem 1.5 and properties of the factor map $p$ under which we obtain $\mathrm{E}(\varphi)=\mathrm{E}(\psi)$.

\section{Preliminaries}

In this section we provide notion and background on topological groups, uniformities, topological dynamical systems, amenable groups, Van Hove nets and uniform lattices.

\subsection{Topological Groups}

Consider a group $G$. We write $e_{G}$ for the neutral element in $G$. For subsets $A, B \subseteq G$ the Minkowski product is defined as $A B:=\{a b ;(a, b) \in A \times B\}$. For $A \subseteq G$ and $g \in G$ we denote $A g:=A\{g\}, g A:=\{g\} A, A^{c}:=G \backslash A$ and the Minkowski inverse $A^{-1}:=$ $\left\{a^{-1} ; a \in A\right\}$. We call $A \subseteq G$ symmetric, if $A=A^{-1}$. In order to omit brackets, we will use the convention, that the inverse and the complement are stronger binding than the Minkowski product, which is stronger binding than the remaining set theoretic operations. Note that the complement and the inverse commute, i.e. $\left(A^{c}\right)^{-1}=\left(A^{-1}\right)^{c}$.

A topological group is a group $G$ equipped with a $T_{1}$-topology ${ }^{6} \tau$, such that the multiplication $\cdot: G \times G \rightarrow G$ and the inverse function $(\cdot)^{-1}: G \rightarrow G$ are continuous. With our definition every topological group is regular, hence Hausdorff, as shown in [31, Theorem 4.8]. An isomorphism of topological groups is a homeomorphism that is a group homomorphism as well. We write $\bar{A}$ for the closure and $\operatorname{int}(A)$ for the interior of a subset $A \subseteq G$. By $\mathcal{K}(G)$ we denote the set of all non-empty compact subsets of $G$.

If $G$ is a locally compact group, a Haar measure on $G$ is a non zero regular Borel measure $\mu$ on $G$, which satisfies $\mu(g A)=\mu(A)=\mu(A g)$ for all $g \in G$ and all Borel sets $A \subseteq G$. $G$ is called unimodular, if it admits a Haar measure. There holds $\mu(U)>0$ for all non empty open $U \subseteq G$ and $\mu(K)<\infty$ for all compact $K \subseteq G$. A Haar measure is unique up to scaling, i.e. if $\mu$ and $v$ are Haar measures on $G$, then there is $c>0$ such that $\mu(A)=c v(A)$ for all Borel measurable sets $A \subseteq G$. If nothing else is mentioned, we denote a Haar measure of a topological group $G$ by $\mu$. If $G$ is discrete we equip $G$ with the counting measure $A \mapsto|A|$, which is a Haar measure. Other examples of unimodular groups are all locally compact

6 A topology is called $T_{1}$, if for any two distinct points $g, g^{\prime} \in G$ there is an open neighbourhood of $g$ that does not contain $g^{\prime}$. 
abelian groups or the Heisenberg group, as presented in Sect. 2.5 below. For reference see $[15,24]$.

\subsection{Compact Hausdorff Uniform Spaces}

Let $X$ be a set. A binary relation on $X$ is a subset of $X \times X$. For binary relations $\eta$ and $\kappa$ on $X$ we denote the inverse $\eta^{-1}:=\{(y, x) ;(x, y) \in \eta\}$, the composition $\eta \kappa:=\{(x, y) ; \exists z \in$ $X:(x, z) \in \eta$ and $(z, y) \in \kappa\}$ and $\eta[x]:=\{y \in X ;(y, x) \in \eta\}$. A binary relation is called symmetric, if $\eta=\eta^{-1}$.

For a compact Hausdorff space $X$, we denote the diagonal $\Delta_{X}:=\{(x, x) ; x \in X\}$ and call a neighbourhood of $\Delta_{X}$ in $X^{2}$ an entourage (of $X$ ). The set of all entourages of $X$ is referred to as the uniformity of $X$ and usually denoted by $\mathbb{U}_{X}$. In this context we refer to $\left(X, \mathbb{U}_{X}\right)$ as a compact Hausdorff uniform space. Note that one can define general "uniform spaces", but as we are only interested in compact Hausdorff spaces, this definition works for us. For details and the general definition we recommend [35]. Note that we obtain our definition to be a restriction of the general definition from [35, Theorem 6.22] and [40, Theorem 32.3]. To obtain some geometric intuition for $\eta \in \mathbb{U}_{X}$ we say that $x$ is $\eta$-close to $y$, whenever $(x, y) \in \eta$. This notion is symmetric iff $\eta$ is symmetric. We think of two elements to be "very close", whenever the pair is $\eta$-close for "many" entourages $\eta$. Note that if $x$ is $\eta$-close to $y$ and $y$ is $\kappa$-close to $z$, then $x$ is $\eta \kappa$-close to $z$.

A subfamily $\mathbb{B}_{X} \subseteq \mathbb{U}_{X}$ is called a base for $\mathbb{U}_{X}$, if every entourage contains a member of $\mathbb{B}_{X}$. An entourage $\eta \in \mathbb{U}_{X}$ is called open (or closed), whenever it is open (or closed) as a subset of $X \times X$. Note that the family of all open and symmetric entourages of $X$ forms a base of the uniformity of $X$. If $(X, d)$ is a metric space we denote $[d<\varepsilon]:=\{(x, y) \in$ $X \times X ; d(x, y)<\varepsilon\}$ for $\varepsilon>0$. Then $\mathbb{B}_{d}:=\{[d<\varepsilon] ; \varepsilon>0\}$ is a base for the uniformity of the corresponding topological space $X$. Note that $x$ is $[d<\varepsilon]$-close to $y$, iff $d(x, y)<\varepsilon$.

\subsection{Actions of a Group on a Topological Space}

Let $G$ be a topological group and $X$ be a topological space. A continuous map $\varphi: G \times X \rightarrow$ $X$ is called an action of $G$ on $X$ (also dynamical system or flow), whenever $\varphi\left(e_{G}, \cdot\right)$ is the identity on $X$ and for all $g, g^{\prime} \in G$ there holds $\varphi\left(g, \varphi\left(g^{\prime}, \cdot\right)\right)=\varphi\left(g g^{\prime}, \cdot\right)$. We write $\varphi^{g}:=\varphi(g, \cdot): X \rightarrow X$ for all $g \in G$. In this context $X$ is called the phase space of the action. If $\varphi$ and $\psi$ are actions of a topological group $G$ on topological spaces $X$ and $Y$ respectively, we call a surjective continuous map $p: X \rightarrow Y$ a factor map, if $p \circ \varphi^{g}=\psi^{g} \circ p$ for all $g \in G$. We then refer to $\psi$ as a factor of $\varphi$ and write $\varphi \stackrel{p}{\rightarrow} \psi$. If $p$ is in addition a homeomorphism, then $p$ is called a topological conjugacy and we call $\varphi$ and $\psi$ topologically conjugate.

\subsection{Amenable Groups and Van Hove Nets}

A partially ordered set $(I, \geq)$ is said to be directed, if $I$ is not empty and if every finite subset of $I$ has an upper bound. A map $f$ from a directed set $I$ to a set $X$ is called a $n e t$ in $X$. We also write $x_{i}$ for $f(i)$ and $\left(x_{i}\right)_{i \in I}$ for $f$. A net $\left(x_{i}\right)_{i \in I}$ in a topological space $X$ is said to converge to $x \in X$, if for every open neighbourhood $U$ of $x$, there exists $j \in I$ such that $x_{i} \in U$ for all $i \geq j$. In this case we also write $\lim _{i \in I} x_{i}=x$. For a net $\left(x_{i}\right)_{i \in I}$ in $\mathbb{R} \cup\{-\infty, \infty\}$, we define $\lim \sup _{i \in I} x_{i}:=\inf _{i \in I} \sup _{j \geq i} x_{j}$ and similarly $\liminf _{i \in I} x_{i}$. Note that $\left(x_{i}\right)_{i \in I}$ converges to 
$x \in \mathbb{R} \cup\{-\infty, \infty\}$, iff there holds $\lim \sup _{i \in I} x_{i}=x=\lim \inf _{i \in I} x_{i}$. For more details, see $[18,35]$.

Let $G$ be a unimodular group. For $K, A \subseteq G$ we define the $K$-boundary of $A$ as

$$
\partial_{K} A:=K \bar{A} \cap K \overline{A^{c}} \text {. }
$$

We use the convention, that the Minkowski operations and the complement are stronger binding than the operation of taking the $K$-boundary and that the set theoretic operations (except from forming the complement) are weaker binding. From the definition we obtain that $K \mapsto \partial_{K} A$ is monotone.

Note that $\partial_{K} A$ is the set of all elements $g \in G$ such that $K^{-1} g$ intersects both $\bar{A}$ and $\overline{A^{c}}$.

Lemma 2.1 For compact subsets $K, L, A \subseteq G$ there holds

(i) $L \partial_{K} A \subseteq \partial_{L K} A$ and $\partial_{K} L A \subseteq \partial_{K L} A$.

(ii) $L A \subseteq A \cup \partial_{L} A$, whenever $e_{G} \in L$.

Proof Straight forward arguments show (ii) and the first statement in (i). To see $\partial_{K} L A \subseteq$ $\partial_{K L} A$ we compute $\overline{(L A)^{c}} \subseteq \overline{(l A)^{c}}=l \overline{A^{c}} \subseteq L \overline{A^{c}}$ for any $l \in L$ and obtain $\partial_{K} L A \subseteq$ $K \overline{L A} \cap K \overline{(L A)^{c}} \subseteq K L \bar{A} \cap K L \overline{A^{c}}=\partial_{K L} A$.

A net $\left(A_{i}\right)_{i \in I}$ of measurable subsets of $G$ is called finally somewhere dense, if there is $j \in I$ such that for all $i \geq j$ the set $A_{i}$ is somewhere dense. ${ }^{7}$ A finally somewhere dense net $\left(A_{i}\right)_{i \in I}$ of compact subsets of $G$ is called a Van Hove net, if for all compact subsets $K \subseteq G$, there holds

$$
\lim _{i \in I} \frac{\mu\left(\partial_{K} A_{i}\right)}{\mu\left(A_{i}\right)}=0
$$

A unimodular group is called amenable whenever it contains a Van Hove net.

Remark 2.2 (i) A finally somewhere dense net $\left(A_{i}\right)_{i \in I}$ of compact sets is a Van Hove net iff for all symmetric compact sets $K$ the Van Hove condition (2) is satisfied. Indeed, if $K$ is an arbitrary non empty but compact set we can choose $k \in K$ and obtain $\partial_{K} A_{i} \subseteq \partial_{k K^{-1} K} A_{i}=k\left(\partial_{K^{-1} K} A_{i}\right)$. As $\mu\left(\partial_{K} A_{i}\right) \leq \mu\left(k\left(\partial_{K^{-1} K} A_{i}\right)\right)=\mu\left(\partial_{K^{-1} K} A_{i}\right)$ holds and $K^{-1} K$ is symmetric and contains $e_{G}$ we obtain the claim.

(ii) A straight forward computation shows $\partial_{K} A=\overline{K A} \backslash\left(\right.$ int $\left.\left(\bigcap_{k \in K} k A\right)\right)$ for $K \subseteq G$ compact and $A \subseteq G$. If we assume in addition $e_{G} \in K=K^{-1}$, then another computation gives $\partial_{K} A=((K \bar{A}) \backslash \operatorname{int}(A)) \cup\left(\left(K^{-1} \overline{A^{c}}\right) \backslash \operatorname{int}\left(A^{c}\right)\right)$. Thus the definitions of the $K$ boundary given above; in [54]; ${ }^{8}$ in [50] and in [23], coincide, whenever $e_{G} \in K=K^{-1}$. As the discussed terms are monotone in $K$, we can adapt the arguments given in (i) to see that all definitions of $K$-boundary yield equivalent definitions of Van Hove nets.

(iii) We call a finally somewhere dense net $\left(A_{i}\right)_{i \in I}$ a Følner net, if for every $g \in G$ there holds $\lim _{i \in I} \mu\left(g A_{i} \Delta A_{i}\right) \mu\left(A_{i}\right)^{-1}=0$, where $A \Delta B:=(A \backslash B) \cup(B \backslash A)$ is the symmetric difference of $A, B \subseteq G$. Følner nets are called "left ergodic nets" in [54]. A net $\left(A_{i}\right)_{i \in I}$ is a Van Hove net, iff it is a Følner net and satisfies $\lim _{i \in I} \mu\left(\partial_{W} A_{i}\right) \mu\left(A_{i}\right)^{-1}=0$ for some neighbourhood $W$ of $e_{G}$ as presented in [54, Appendix; (3.K)]. From this we obtain that every Van Hove net is a Følner net and that the notions of Van Hove and Følner nets are equivalent for discrete groups. Note that our definition of $K$-boundary and of Van Hove nets is inspired from [36], where it is used to define Følner nets in discrete amenable groups. In [54, Appendix; Example 3.4] a Følner net in $\mathbb{R}^{d}$ is presented, that is not a Van Hove net.

\footnotetext{
$7 A \subseteq G$ is called somewhere dense if it has nonempty interior. Note that this ensures $\mu(A)>0$.

8 Note that in [54] the order of multiplication is inverse to our notation.
} 
(iv) It is shown for $\sigma$-compact locally compact groups in [54, Appendix 3.L] and for second countable unimodular groups in [47, Lemma 2.7] that the existence of Van Hove sequences is equivalent to the existence of Følner sequences. The corresponding arguments generalize to unimodular groups without countability assumptions if we consider nets instead of sequences. For further equivalent notions of amenability we recommend the monographs $[45,46]$.

Proposition 2.3 Let $K, C \subseteq G$ be nonempty and compact subsets and $\left(A_{i}\right)_{i \in I}$ be a Van Hove net in $G$. Then $\left(K A_{i}\right)_{i \in I}$ and $\left(C A_{i}\right)_{i \in I}$ are Van Hove nets and satisfy $\lim _{i \in I} \frac{\mu\left(K A_{i}\right)}{\mu\left(C A_{i}\right)}=1$.

Proof It clearly suffices to consider the case $C=\left\{e_{G}\right\}$. Let $L \subseteq G$ be compact.

As $L K$ is compact, we obtain $\left(K A_{i}\right)_{i \in I}$ to be a Van Hove net from

$$
0 \leq \frac{\mu\left(\partial_{L} K A_{i}\right)}{\mu\left(K A_{i}\right)} \leq \frac{\mu\left(\partial_{L K} A_{i}\right)}{\mu\left(A_{i}\right)} \stackrel{i \in I}{\rightarrow} 0 .
$$

To show $\lim _{i \in I} \frac{\mu\left(K A_{i}\right)}{\mu\left(A_{i}\right)}=1$ let $k \in K^{-1}$ and note that $k K A_{i} \subseteq A_{i} \cup \partial_{k K} A_{i}$ implies

$$
1 \leq \frac{\mu\left(K A_{i}\right)}{\mu\left(A_{i}\right)}=\frac{\mu\left(k K A_{i}\right)}{\mu\left(A_{i}\right)} \leq 1+\frac{\mu\left(\partial_{k K} A_{i}\right)}{\mu\left(A_{i}\right)} \stackrel{i \in I}{\rightarrow} 1 .
$$

\subsection{Uniform Lattices in Locally Compact Topological Groups}

Let $G$ be a locally compact topological group. A discrete subgroup $\Lambda \subseteq G$ is called a uniform lattice, whenever it is co-compact, i.e. whenever $G / \Lambda$ is compact. A fundamental domain of $\Lambda$ is a subset $C \subseteq G$ such that each element of $G$ can be written in a unique way as $g=c z$ with $c \in C$ and $z \in \Lambda$.

Remark 2.4 (i) Every uniform lattice allows the choice of a pre-compact ${ }^{9}$ and Borel measurable fundamental domain $C$ such that $e_{G} \in C$ and such that $0<\mu(C) \leq \mu(\bar{C})<\infty$. Whenever we consider a fundamental domain we will assume such a choice. To see that such a choice is possible consider a pre-compact and open neighbourhood of $e_{G}$ such that $U^{-1} U \cap \Lambda=\left\{e_{G}\right\}$ and a compact set $K \subseteq G$ such that $K \Lambda=G$. Without lost of generality we assume $e_{G} \in K$. Let furthermore $\left(k_{n}\right)_{n=1}^{N}$ be a finite sequence in $K$ such that $k_{1}=e_{G}$ and such that $\bigcup_{n=1}^{N}\left(k_{n} U\right) \supseteq K$. Then

$$
C:=\bigcup_{n=1}^{N}\left[\left(k_{n} U\right) \backslash\left(\bigcup_{i<n} k_{i} U \Lambda\right)\right]
$$

satisfies the considered properties.

(ii) By [15, Theorem 9.1.6] every locally compact group that contains a uniform lattice is unimodular. If $F \subseteq \Lambda$ is finite, then the finite union $\bigcup_{z \in F} C z$ is disjoint and measurable. Thus by the right invariance of the Haar measure there holds

$$
\mu(C F)=\sum_{z \in F} \mu(C z)=\mu(C)|F|
$$

\footnotetext{
${ }^{9}$ A subset $A$ of a topological space $X$ is called pre-compact, whenever the closure $\bar{A}$ is compact in $X$.
} 
Example 2.5 (i) If $H$ is a compact abelian group and $a, b \in \mathbb{N}$, then $\mathbb{R}^{a} \times \mathbb{Z}^{b} \times H$ contains the countable uniform lattice $\mathbb{Z}^{a+b} \times\left\{e_{H}\right\}$ with fundamental domain $[0,1)^{a} \times\{0\}^{b} \times H$. Note that up to isomorphism these are all compactly generated locally compact abelian groups $G$. [31, Theorem 9.8].

(ii) The Heisenberg group $H_{3}(\mathbb{R}):=\left\{\left(\begin{array}{lll}1 & a & c \\ 0 & 1 & b \\ 0 & 0 & 1\end{array}\right) ; a, b, c \in \mathbb{R}\right\}$ under matrix multiplication is a non-abelian amenable group and contains the uniform lattice $H_{3}(\mathbb{Z})$ with fundamental domain $H_{3}([0,1))$. For reference see [22, Example 2.13] and [48, Exercise $1.2 .4]$.

\section{The Ornstein-Weiss Lemma}

In the introduction we defined what it means that a group satisfies the Ornstein-Weiss lemma. From [36, Theorem 1.1.] or [13, Theorem 1.1] we know that every discrete amenable group satisfies the Ornstein-Weiss lemma. Thus whenever a group $G$ contains a uniform lattice $\Lambda$, we know that the Ornstein-Weiss lemma holds in $\Lambda$. After a short remark on the history of the Ornstein-Weiss lemma we show that this implies that the Ornstein-Weiss lemma holds for $G$ as well, i.e. Theorem 1.1.

Remark 3.1 For the origins of the ideas of a proof of the Ornstein-Weiss lemma in countable amenable groups see [43,57] and in particular [29, 1.3.1]. These ideas are furthermore considered in $[16,33,37]$. The corresponding arguments are worked out in detail for discrete amenable groups and even for discrete amenable semigroups in [13,36,58]. In the last part of the arguments presented in [29] one uses that $\sup _{D \in \mathcal{K}(G)} f(D) / \mu(D)<\infty$ and in particular that this boundedness holds for $D \in \mathcal{K}(G)$ with small Haar measure, which are "spread out" a lot over $G$. See [36] for detail. In the discrete case $f(M) \leq|M| f\left(\left\{e_{G}\right\}\right)$ follows easily from the right invariance and the subadditivity. Note that adding this relative boundedness to the assumptions on $f$ it is shown in [47] that a modified version of the Ornstein-Weiss lemma remains valid also for the groups considered in our context. However this boundedness assumption is not satisfied for the functions which are considered in the definition of topological entropy as we discuss in Remark 4.6(iv) below.

\subsection{Extrapolation from a Uniform Lattice}

We will first construct Van Hove nets in a uniform lattice $\Lambda \subseteq G$ from Van Hove nets in $G$ with properties that allow to extract the validity of the Ornstein-Weiss lemma from the lattice.

Lemma 3.2 Let $G$ be an amenable group and $\Lambda$ be a uniform lattice in $G$ with fundamental domain $C$. Then for every Van Hove net $\left(A_{i}\right)_{i \in I}$ in $G$ there exist Van Hove nets $\left(\breve{F}_{i}\right)_{i \in I}$ and $\left(\hat{F}_{i}\right)_{i \in I}$ in $\Lambda$ that satisfy

(i) $C \check{F}_{i} \subseteq A_{i} \subseteq C \hat{F}_{i}$ for all $i \in I$ and

(ii) $\lim _{i \in I} \frac{\left|\hat{F}_{i}\right|}{\left|\check{F}_{i}\right|}=1$.

Proof For $i \in I$ let $\left(A_{i}\right)_{i \in I}$ be a Van Hove net in $G$. Set $\check{F}_{i}:=\left\{z \in \Lambda ; C z \subseteq A_{i}\right\}$ and $\hat{F}_{i}:=\left\{z \in \Lambda ; C z \cap A_{i} \neq \emptyset\right\}$. Then (i) follows directly from these definitions. In order to 
simplify the notation let $\check{A}_{i}:=C \check{F}_{i}$ and $\hat{A}_{i}:=C \hat{F}_{i}$ for $i \in I$. Let furthermore $K:=\overline{C C^{-1}}$ and note that $e_{G} \in K=K^{-1}$. The complements and boundaries in this proof are taken with respect to $G$ unless otherwise mentioned. For $z \in \Lambda$ we know that $z \notin C^{-1} A_{i}^{c}$ is equivalent to $C z \cap A_{i}^{c}=\emptyset$ and thus obtain $\check{F}_{i}=\Lambda \backslash\left(C^{-1} A_{i}^{c}\right)$. A similar argument yields $\hat{F}_{i}=\Lambda \cap C^{-1} A_{i}$.

Before we show that $\left(\check{F}_{i}\right)_{i \in I}$ and $\left(\hat{F}_{i}\right)_{i \in I}$ are Van Hove nets in $\Lambda$ we will show that the ratio of their cardinalities tends to 1 , i.e. (ii). We compute $\hat{A}_{i}=C\left(\Lambda \cap C^{-1} A_{i}\right) \subseteq C \Lambda \cap K A_{i}=$ $K A_{i}$ and similarly $\left(\check{A}_{i}\right)^{c}=C\left(\Lambda \backslash \check{F}_{i}\right)=C\left(\Lambda \cap C^{-1} A_{i}^{c}\right) \subseteq K A_{i}^{c}$, from which we obtain $\overline{\hat{A}_{i}} \cap \overline{\check{A}_{i}^{c}} \subseteq K \overline{A_{i}} \cap K \overline{A_{i}^{c}}=\partial_{K} A_{i}$. Thus for all $i \in I$ there holds

$$
\overline{\hat{A}_{i}} \subseteq \partial_{K} A_{i} \cup \check{A}_{i} \text { and } \overline{\check{A}_{i}^{c}} \subseteq \partial_{K} A_{i} \cup \hat{A}_{i}^{c}
$$

We will need both inclusions later in this proof, but for now obtain from the first one that $A_{i} \subseteq \hat{A}_{i} \subseteq \check{A}_{i} \cup \partial_{K} A_{i}$. Hence $\mu\left(\hat{A}_{i}\right) \leq \mu\left(\check{A}_{i}\right)+\mu\left(\partial_{K} A_{i}\right)$ and $\mu\left(A_{i}\right)-\mu\left(\partial_{K} A_{i}\right) \leq \mu\left(\check{A}_{i}\right)$. As $\lim _{i \in I} \frac{\mu\left(A_{i}\right)}{\mu\left(\partial_{K} A_{i}\right)}=\infty$ we get (ii) from the computation

$$
\begin{aligned}
1 & \leq \frac{\left|\hat{F}_{i}\right|}{\left|\check{F}_{i}\right|}=\frac{\mu\left(\hat{A}_{i}\right)}{\mu\left(\check{A}_{i}\right)} \leq \frac{\mu\left(\check{A}_{i}\right)+\mu\left(\partial_{K} A_{i}\right)}{\mu\left(\check{A}_{i}\right)}=1+\frac{\mu\left(\partial_{K} A_{i}\right)}{\mu\left(\check{A}_{i}\right)} \\
& \leq 1+\frac{\mu\left(\partial_{K} A_{i}\right)}{\mu\left(A_{i}\right)-\mu\left(\partial_{K} A_{i}\right)}=1+\frac{1}{\frac{\mu\left(A_{i}\right)}{\mu\left(\partial_{K} A_{i}\right)}-1} .
\end{aligned}
$$

We will finish the proof by showing that $\left(\check{F}_{i}\right)_{i \in I}$ and $\left(\hat{F}_{i}\right)_{i \in I}$ are Van Hove nets. To do this consider a finite set $F \in \Lambda$ and set $L:=\overline{C F}$. From (ii) we know the existence of $j \in I$ such that for all $i \geq j$ there holds

$$
1 \leq \frac{\mu\left(\hat{A}_{i}\right)}{\mu\left(\check{A}_{i}\right)}=\frac{\left|\hat{F}_{i}\right|}{\left|\check{F}_{i}\right|} \leq 2 .
$$

Hence $\mu\left(\check{A}_{i}\right) \leq \mu\left(A_{i}\right) \leq \mu\left(\hat{A}_{i}\right) \leq 2 \mu\left(\check{A}_{i}\right)$. From (3) and $\check{A}_{i} \subseteq \overline{A_{i}}$ we obtain furthermore

$$
L \overline{\hat{A}_{i}} \subseteq L\left(\partial_{K} A_{i} \cup \overline{A_{i}}\right) \subseteq L \partial_{K} A_{i} \cup L \overline{A_{i}} \subseteq \partial_{L K} A_{i} \cup L \overline{A_{i}}
$$

and analogously $L \overline{L \check{A}_{i}^{c}} \subseteq \partial_{L K} A_{i} \cup L \overline{A_{i}^{c}}$. As there also holds $L \overline{\hat{A}_{i}^{c}} \subseteq L \overline{A_{i}^{c}} \subseteq \partial_{L K} A_{i} \cup L \overline{A_{i}^{c}}$ and similarly $\overline{L \check{A}_{i}} \subseteq \partial_{L K} A_{i} \cup L \overline{A_{i}}$ we obtain from $L \subseteq L K$ that

$$
\begin{aligned}
\partial_{L} \hat{A}_{i} \cup \partial_{L} \check{A}_{i} & =\left(L \overline{\hat{A}_{i}} \cap L \overline{\hat{A}_{i}^{c}}\right) \cup\left(L \overline{\check{A}_{i}} \cap L \overline{\check{A}_{i}^{c}}\right) \\
& \subseteq\left(\partial_{L K} A_{i} \cup L \overline{A_{i}}\right) \cap\left(\partial_{L K} A_{i} \cup L \overline{A_{i}^{c}}\right) \\
& =\partial_{L K} A_{i} \cup\left(L \overline{A_{i}} \cap L \overline{A_{i}^{c}}\right)=\partial_{L K} A_{i}
\end{aligned}
$$

We thus obtain for all $i \geq j$ that

$$
\frac{\mu\left(\partial_{L} \hat{A}_{i}\right)}{\mu\left(\hat{A}_{i}\right)} \leq \frac{\mu\left(\partial_{L K} A_{i}\right)}{\mu\left(A_{i}\right)} \text { and } \frac{\mu\left(\partial_{L} \check{A}_{i}\right)}{\mu\left(\check{A}_{i}\right)} \leq 2 \frac{\mu\left(\partial_{L K} A_{i}\right)}{\mu\left(A_{i}\right)}
$$


Denoting the $F$-boundary taken of a subset $E$ with respect to $\Lambda$ or $G$ by $\partial_{F}^{\Lambda} E$ or $\partial_{F}^{G} E$, respectively, we use that $C$ is a fundamental domain to get

$$
\begin{aligned}
\partial_{F}^{\Lambda} \check{F}_{i} & =F \check{F}_{i} \cap F\left(\Lambda \backslash \check{F}_{i}\right) \subseteq F C \check{F}_{i} \cap F C\left(\Lambda \backslash \check{F}_{i}\right) \\
& =F C \check{F}_{i} \cap F\left(G \backslash C \check{F}_{i}\right) \subseteq F C \bar{F} \check{F}_{i} \cap F \overline{G \backslash C \check{F}_{i}}=\partial_{F}^{G} \check{A}_{i},
\end{aligned}
$$

hence $C \partial_{F}^{\Lambda}\left(\check{F}_{i}\right) \subseteq C \partial_{F}^{G} \check{A}_{i} \subseteq \partial_{C F}^{G} \check{A}_{i} \subseteq \partial_{L}^{G} \check{A}_{i}$. Thus, considering (4) we compute

$$
0 \leq \frac{\left|\partial_{F}^{\Lambda} \check{F}_{i}\right|}{\left|\check{F}_{i}\right|}=\frac{\mu\left(C \partial_{F}^{\Lambda} \check{F}_{i}\right)}{\mu\left(\check{A}_{i}\right)} \leq \frac{\mu\left(\partial_{L}^{G} \check{A}_{i}\right)}{\mu\left(\check{A}_{i}\right)} \leq 2 \frac{\mu\left(\partial_{L K}^{G} A_{i}\right)}{\mu\left(A_{i}\right)} .
$$

As $L K$ is compact and $\left(A_{i}\right)_{i \in I}$ is a Van Hove net in $G$ we obtain $\left(\check{F}_{i}\right)_{i \in I}$ to be a Van Hove net in $\Lambda$. Similarly one shows $\left(\hat{F}_{i}\right)_{i \in I}$ to be a Van Hove net.

From the next theorem we obtain that every amenable group that contains a uniform lattice satisfies the Ornstein-Weiss lemma, i.e. the statement of Theorem 1.1.

Theorem 3.3 If $f: \mathcal{K}(G) \rightarrow \mathbb{R}$ is a subadditive, right invariant and monotone function and $\left(A_{i}\right)_{i \in I}$ is a Van Hove net in $G$, then

$$
\lim _{i \in I} \frac{f\left(A_{i}\right)}{\mu\left(A_{i}\right)}=\frac{1}{\mu(C)} \lim _{j \in J} \frac{f\left(\bar{C} F_{j}\right)}{\left|F_{j}\right|}
$$

holds for any Van Hove net $\left(F_{j}\right)_{j \in J}$ in a uniform lattice $\Lambda \subseteq G$ with fundamental domain $C$.

Proof Let $C$ be a fundamental domain of $\Lambda$ in $G$ and note that $\mathcal{K}(\Lambda)$ is the set of finite subsets of $\Lambda$. In order to use that every discrete amenable group satisfies the Ornstein-Weiss lemma, we define

$$
f^{\Lambda}: \mathcal{K}(\Lambda) \rightarrow \mathbb{R} ; F \mapsto f(\bar{C} F)
$$

It is straight forward to see, that $f^{\Lambda}$ is right invariant and monotone. In order to show, that $f^{\Lambda}$ is subadditive let $F, F^{\prime} \in \mathcal{K}(\Lambda)$. As $\bar{C}\left(F \cup F^{\prime}\right) \subseteq \bar{C} F \cup \bar{C} F^{\prime}$ we obtain from the monotonicity and the subadditivity of $f$ that

$$
f^{\Lambda}\left(F \cup F^{\prime}\right) \leq f\left(\bar{C} F \cup \bar{C} F^{\prime}\right) \leq f^{\Lambda}(F)+f^{\Lambda}\left(F^{\prime}\right) .
$$

Let now $\left(A_{i}\right)_{i \in I}$ be a Van Hove net in $G$ and $\left(F_{j}\right)_{j \in J}$ be a Van Hove net in $\Lambda$. By Lemma 3.2 there are Van Hove nets $\left(\check{F}_{i}\right)_{i \in I}$ and $\left(\hat{F}_{i}\right)_{i \in I}$ such that $C \check{F}_{i} \subseteq A_{i} \subseteq C \hat{F}_{i}$ for all $i \in I$ and $\lim _{i \in I}\left|\hat{F}_{i}\right|\left|\check{F}_{i}\right|^{-1}=1$. As $A_{i}$ is closed, we get furthermore $\bar{C} \check{F}_{i} \subseteq A_{i} \subseteq \bar{C} \hat{F}_{i}$ and hence

$$
f^{\Lambda}\left(\check{F}_{i}\right) \leq f\left(A_{i}\right) \leq f^{\Lambda}\left(\hat{F}_{i}\right) .
$$

Note that $\Lambda$ is a discrete amenable group by Lemma 3.2 and thus satisfies the Ornstein-Weiss lemma. This implies the existence of the following limits and

$$
\lim _{i \in I} \frac{f^{\Lambda}\left(\check{F}_{i}\right)}{\left|\check{F}_{i}\right|}=\lim _{i \in I} \frac{f^{\Lambda}\left(\hat{F}_{i}\right)}{\left|\hat{F}_{i}\right|}=\lim _{j \in J} \frac{f^{\Lambda}\left(F_{j}\right)}{\left|F_{j}\right|} .
$$

Let $\varepsilon>0$. As $\lim _{i \in I}\left|\hat{F}_{i}\right|\left|\check{F}_{i}\right|^{-1}=1$ and $\left|\check{F}_{i}\right| \leq\left|\hat{F}_{i}\right|$ for all $i \in I$ there is $j \in I$, such that for all $i \geq j$ there holds $\left|\hat{F}_{i}\right| \leq(1+\varepsilon)\left|\breve{F}_{i}\right|$ and hence $\frac{1}{(1+\varepsilon)}\left|\hat{F}_{i}\right| \mu(C) \leq \mu(C)\left|\check{F}_{i}\right|=\mu\left(C \check{F}_{i}\right) \leq \mu\left(A_{i}\right) \leq \mu\left(C \hat{F}_{i}\right)=\mu(C)\left|\hat{F}_{i}\right| \leq(1+\varepsilon)\left|\check{F}_{i}\right| \mu(C)$. 
Thus for $j \geq i$ there holds

$$
\frac{1}{(1+\varepsilon)} \frac{f^{\Lambda}\left(\check{F}_{i}\right)}{\left|\check{F}_{i}\right|} \leq \mu(C) \frac{f\left(A_{i}\right)}{\mu\left(A_{i}\right)} \leq(1+\varepsilon) \frac{f^{\Lambda}\left(\hat{F}_{i}\right)}{\left|\hat{F}_{i}\right|} .
$$

We obtain for every $\varepsilon>0$ that

$$
\frac{1}{(1+\varepsilon)} \lim _{i \in I} \frac{f^{\Lambda}\left(\check{F}_{i}\right)}{\left|\check{F}_{i}\right|} \leq \mu(C) \liminf _{i \in I} \frac{f\left(A_{i}\right)}{\mu\left(A_{i}\right)} \leq \mu(C) \limsup _{i \in I} \frac{f\left(A_{i}\right)}{\mu\left(A_{i}\right)} \leq(1+\varepsilon) \lim _{i \in I} \frac{f^{\Lambda}\left(\hat{F}_{i}\right)}{\left|\hat{F}_{i}\right|} .
$$

This shows that the limit $\mu(C) \lim _{i \in I} \frac{f\left(A_{i}\right)}{\mu\left(A_{i}\right)}$ exists and that it equals the limits in (5).

In particular it does not depend on the choice of $\left(A_{i}\right)_{i \in I}$.

If $(G, H, \Lambda)$ is a CPS we know that $G \times H$ contains a uniform lattice and in order to show that $G$ satisfies the Ornstein-Weiss lemma one could hope that the properties of a CPS also imply that $G$ contains a uniform lattice. The next example was already studied in [39] and shows that there are CPS with a physical space that contains no uniform lattices.

Example 3.4 Consider the additive group of the $p$-adic numbers $\mathbb{Q}_{p}$. For reference on $p$-adic numbers see [8, Example 2.10] and [28]. Denote by $\mathbb{Z}\left[p^{-1}\right]$ the smallest subring of $\mathbb{Q}_{p}$ (or $\mathbb{R})$ that contains $\mathbb{Z}$ and $p^{-1}$. Then $\left(\mathbb{Q}_{p}, \mathbb{R}, \Lambda\right)$ with $\Lambda:=\left\{(x, x) ; x \in \mathbb{Z}\left[p^{-1}\right]\right\}$ is a cut and project scheme. Furthermore the only discrete subgroup of $\mathbb{Q}_{p}$ is $\{0\}$, which is not cocompact. Thus $\mathbb{Q}_{p}$ does not contain a uniform lattice. For reference on this example see [12, Example 5.C.10(2)] and [39, Chapter II.10].

Nevertheless we can obtain all physical spaces of CPS to satisfy the Ornstein-Weiss lemma from the following result, which we already stated in Theorem 1.2.

Theorem 3.5 If $G$ and $H$ are amenable groups such that $G \times H$ satisfies the Ornstein-Weiss lemma, then $G$ and $H$ satisfy the Ornstein-Weiss lemma.

To prove this theorem we need the following.

Lemma 3.6 Let $G$ and $H$ be unimodular groups and assume that $\left(A_{i}\right)_{i \in I}$ and $\left(B_{j}\right)_{j \in J}$ are Van Hove nets in $G$ and $H$ respectively. Then $\left(A_{i} \times B_{j}\right)_{(i \times j) \in I \times J}$ is a Van Hove net in $G \times H$, where $I \times J$ is ordered component wise.

Proof Consider a compact subset $M \subseteq G \times H$ and let $K:=\pi_{G}(M)$ and $C:=\pi_{H}(M)$ the projections. A straight forward computation shows

$$
\partial_{M}\left(A_{i} \times B_{j}\right) \subseteq\left(\partial_{K} A_{i} \times \partial_{C} B_{j}\right) \cup\left(K A_{i} \times \partial_{C} B_{j}\right) \cup\left(\partial_{K} A_{i} \times C B_{j}\right),
$$

for all $i \in I$ and $j \in J$. Thus Proposition 2.3 yields

$$
\begin{aligned}
0 & \leq \frac{\mu_{G} \times \mu_{H}\left(\partial_{M}\left(A_{i} \times B_{j}\right)\right)}{\mu_{G} \times \mu_{H}\left(A_{i} \times B_{j}\right)} \\
& \leq \frac{\mu_{G}\left(\partial_{K} A_{i}\right) \mu_{H}\left(\partial_{C} B_{j}\right)}{\mu_{G}\left(A_{i}\right) \mu_{H}\left(B_{j}\right)}+\frac{\mu_{G}\left(K A_{i}\right) \mu_{H}\left(\partial_{C} B_{j}\right)}{\mu_{G}\left(A_{i}\right) \mu_{H}\left(B_{j}\right)}+\frac{\mu_{G}\left(\partial_{K} A_{i}\right) \mu_{H}\left(C B_{j}\right)}{\mu_{G}\left(A_{i}\right) \mu_{H}\left(B_{j}\right)} \stackrel{(i, j) \in I \times J}{\longrightarrow} 0 .
\end{aligned}
$$

Proof of Theorem 3.5 Clearly $G$ and $H$ are amenable groups as the projections of the amenable group $G \times H$. Consider a monotone right-invariant and subadditive mapping $f: \mathcal{K}(G) \rightarrow \mathbb{R}$ and a Van Hove net $\left(A_{i}\right)_{i \in I}$. Denote by $\mu_{H}$ the Haar measure of $H$ and choose the Haar 
measure $\mu_{G \times H}$ as the product measure $\mu_{G} \times \mu_{H}$. Let $\left(B_{j}\right)_{j \in J}$ be any Van Hove net in $H$. It is easy to see that $h: \mathcal{K}(G \times H) \rightarrow \mathbb{R}$ defined by

$$
h(Q):=\inf \left\{\sum_{n=1}^{N} f\left(C_{n}\right) \mu_{H}\left(D_{n}\right) ; N \in \mathbb{N}, C_{n} \subseteq G, D_{n} \subseteq H, Q \subseteq \bigcup_{n=1}^{N} C_{n} \times D_{n}\right\}
$$

is monotone, right invariant and subadditive. We next show that for compact subsets $A \subseteq G$ and $B \subseteq H$ there holds $h(A \times B)=f(A) \mu_{H}(B)$. Clearly there holds $h(A \times B) \leq$ $f(A) \mu_{H}(B)$. To show the other inequality let $C_{n} \subseteq G$ and $D_{n} \subseteq H$ such that $A \times B \subseteq$ $\bigcup_{n=1}^{N} C_{n} \times D_{n}$. Without lost of generality we assume $D_{n} \subseteq B$ for $n \in \mathcal{N}:=\{1, \ldots, N\}$. Let furthermore $\left\{E_{1}, \ldots, E_{M}\right\}$ be a finite Borel partition of $B$ s.t. $\bigcup_{m \in \mathcal{M}_{n}} E_{m}=D_{n}$ for all $n \in \mathcal{N}$, where we denote $\mathcal{M}:=\{1, \ldots, M\}$ and $\mathcal{M}_{n}:=\left\{m^{\prime} \in \mathcal{M} ; E_{m^{\prime}} \subseteq D_{n}\right\}$. Setting $\mathcal{N}_{m}:=\left\{n^{\prime} \in \mathcal{N} ; E_{m} \subseteq D_{n^{\prime}}\right\}$ one obtains $A \subseteq \bigcup_{n \in \mathcal{N}_{m}} C_{n}$ and thus by the subadditivity of $f$ that $f(A) \leq \sum_{n \in \mathcal{N}_{m}} f\left(C_{n}\right)$ for all $m \in \mathcal{M}$. Hence

$$
\begin{aligned}
\sum_{n \in \mathcal{N}} f\left(C_{n}\right) \mu_{H}\left(D_{n}\right) & =\sum_{n \in \mathcal{N}} \sum_{m \in \mathcal{M}_{n}} f\left(C_{n}\right) \mu_{H}\left(E_{m}\right)=\sum_{n \in \mathcal{N}, m \in \mathcal{M}, E_{m} \subseteq D_{n}} f\left(C_{n}\right) \mu_{H}\left(E_{m}\right) \\
& =\sum_{m \in \mathcal{M}} \sum_{n \in \mathcal{N}_{m}} f\left(C_{n}\right) \mu_{H}\left(E_{m}\right) \geq \sum_{m \in \mathcal{M}} f(A) \mu_{H}\left(E_{m}\right)=f(A) \mu_{H}(B) .
\end{aligned}
$$

This shows $h(A \times B)=f(A) \mu_{H}(B)$ for compact subsets $A \subseteq G$ and $B \subseteq H$. By Lemma 3.6 we obtain that $\left(A_{i} \times B_{j}\right)_{(i, j) \in I \times J}$ is a Van Hove net in $G \times H$. As $G \times H$ satisfies the Ornstein-Weiss lemma this implies that

$$
\frac{f\left(A_{i}\right)}{\mu_{G}\left(A_{i}\right)}=\frac{f\left(A_{i}\right) \mu_{H}\left(B_{j}\right)}{\mu_{G}\left(A_{i}\right) \mu_{H}\left(B_{j}\right)}=\frac{h\left(A_{i} \times B_{j}\right)}{\mu_{G \times H}\left(A_{i} \times B_{j}\right)},
$$

converges to a limit independent from $\left(A_{i}\right)_{i \in I}$ and we obtain that $G$ satisfies the Ornstein Weiss lemma.

\section{Basics of Entropy Theory}

During this section let $G$ be an amenable group that satisfies the Ornstein-Weiss lemma.

\subsection{Bowen Entourage}

For an action $\varphi: G \times X \rightarrow X$ on a compact Hausdorff space, an entourage $\eta \in \mathbb{U}_{X}$ and a compact subset $A \subseteq G$ we define the Bowen entourage as

$$
\eta_{A}:=\left\{(x, y) ; \forall g \in A:\left(\varphi^{g}(x), \varphi^{g}(y)\right) \in \eta\right\}=\bigcap_{g \in A}\left(\varphi^{g} \times \varphi^{g}\right)^{-1}(\eta) .
$$

We will show in Lemma 4.2 below that the Bowen entourage are indeed entourages of $X$. In order to omit brackets we will use the convention, that the operation of taking a Bowen entourage is a stronger operation than the product of entourages.

Remark 4.1 It is straight forward to show, that $d_{A}$ is a metric and that $\left[d_{A}<\varepsilon\right]=[d<\varepsilon]_{A}$ for all compact $A \subseteq G$ and $\varepsilon>0$.

Lemma 4.2 Let $\varphi: G \times X \rightarrow X$ be a flow on a compact Hausdorff space. For every $\eta \in \mathbb{U}_{X}$ and every compact subset $A \subseteq G$ there holds $\eta_{A} \in \mathbb{U}_{X}$. 
Proof Note that $\varphi: A \times X \rightarrow X$ is a continuous mapping from a compact Hausdorff space. Thus by [35, Theorem 6.31] $(\varphi \times \varphi)^{-1}(\eta)$ is contained in the uniformity of $A \times X$. For $\kappa \in \mathbb{U}_{A}$ and $\vartheta \in \mathbb{U}_{X}$ we set $\kappa \overline{\times} \vartheta:=\left\{\left(g, x, g^{\prime}, x^{\prime}\right) \in(A \times X) \times(A \times X) ;\left(g, g^{\prime}\right) \in\right.$ $\kappa$ and $\left.\left(x, x^{\prime}\right) \in \vartheta\right\}$. As $\left\{\kappa \overline{\times} \vartheta ; \kappa \in \mathbb{U}_{A}\right.$ and $\left.\vartheta \in \mathbb{U}_{X}\right\}$ is a base for the product uniformity on $A \times X$ there are $\kappa \in \mathbb{U}_{A}$ and $\vartheta \in \mathbb{U}_{X}$ with

$$
\kappa \overline{\times} \vartheta \subseteq(\varphi \times \varphi)^{-1}(\eta)=\left\{\left(g, x, g^{\prime}, x^{\prime}\right) \in(A \times X) \times(A \times X) ;\left(\varphi^{g}(x), \varphi^{g^{\prime}}\left(x^{\prime}\right)\right) \in \eta\right\} .
$$

For $\left(x, x^{\prime}\right) \in \vartheta$ and $g \in A$ there holds $\left(g, x, g, x^{\prime}\right) \in \kappa \overline{\times} \vartheta$ and we obtain $\left(\varphi^{g}(x), \varphi^{g}(y)\right) \in \eta$. This proves $\vartheta \subseteq \eta_{A}$ and hence $\eta_{A} \in \mathbb{U}_{X}$.

The following is straight forward to prove and justifies to write $\eta_{A B}$ for $\eta_{(A B)}=\left(\eta_{A}\right)_{B}$.

Proposition 4.3 For $\eta, \kappa \in \mathbb{U}_{X}$ and compact subsets $A, B \subseteq G$ there holds $\eta_{(A B)}=\left(\eta_{A}\right)_{B}$, $\eta_{A \cup B}=\eta_{A} \cap \eta_{B}$ and $\eta_{A} \kappa_{A} \subseteq(\eta \kappa)_{A}$.

\subsection{Relative Topological Entropy}

The following approach to relative topological entropy is inspired by the approach to topological entropy of $\mathbb{Z}$-actions on compact metric spaces via sets of small diameter, given in [11, Sect. 2.5]. Consider first a compact Hausdorff space $X$ and $\eta \in \mathbb{U}_{X}$.

Definition 4.4 For $\eta \in \mathbb{U}_{X}$ we say that a subset $M \subseteq X$ is $\eta$-small, if any $x \in M$ is $\eta$-close to any $y \in M$, i.e. iff $M^{2} \subseteq \eta$. We say, that a set $\mathcal{U}$ of subsets of $X$ is of scale $\eta$, if $U$ is $\eta$-small for every $U \in \mathcal{U}$. As $X$ is compact there is a finite open cover of $X$ of scale $\eta$. Thus for every $M \subseteq X$ there exists a finite open cover of scale $\eta$ as well. For $M \subseteq X$ and $\eta \in \mathbb{U}_{X}$ we denote by $\operatorname{cov}_{M}(\eta)$ the minimal cardinality of an open cover of $M$ of scale $\eta$. If $p: X \rightarrow Y$ is a map to some set $Y$, we define

$$
\operatorname{cov}_{p}(\eta):=\sup _{y \in Y} \operatorname{cov}_{p^{-1}(y)}(\eta) .
$$

A well known $\operatorname{argument}$ shows that $\mathcal{K}(G) \ni A \mapsto \log \left(\operatorname{cov}_{p}\left(\eta_{A}\right)\right)$ is monotone, right invariant and subadditive for every $\eta \in \mathbb{U}_{X}$. Thus the limit in the following definition of relative topological entropy exists and is independent from the choice of a Van Hove net.

Definition 4.5 Let $\varphi$ be an action of $G$ on a compact Hausdorff space $X$ and $\psi$ be a factor of $\varphi$ via factor map $p$. For some Van Hove net $\left(A_{i}\right)_{i \in I}$ and $\eta \in \mathbb{U}_{X}$, we define

$$
\mathrm{E}(\eta \mid \varphi \stackrel{p}{\rightarrow} \psi):=\lim _{i \in I} \frac{\log \left(\operatorname{cov}_{p}\left(\eta_{A_{i}}\right)\right)}{\mu\left(A_{i}\right)} .
$$

We furthermore define the relative topological entropy of $\varphi$ relative to $\psi$ as

$$
\mathrm{E}(\varphi \stackrel{p}{\rightarrow} \psi):=\sup _{\eta \in \mathbb{U}_{X}} \mathrm{E}(\eta \mid \varphi \stackrel{p}{\rightarrow} \psi) .
$$

The topological entropy of $\varphi$ is defined as the relative topological entropy relative to the one point flow. Note that in this case $\operatorname{cov}_{p}(\eta)=\operatorname{cov}_{X}(\eta)$ is the minimal cardinality of an open covering of $X$ of scale $\eta$.

Remark 4.6 (i) There holds $\mathrm{E}(\varphi \stackrel{p}{\rightarrow} \psi)=\sup _{\eta \in \mathbb{B}_{X}} \mathrm{E}(\eta \mid \varphi \stackrel{p}{\rightarrow} \psi)$ for any base $\mathbb{B}_{X}$ of $\mathbb{U}_{X}$, as $\mathrm{E}(\eta \mid \varphi \stackrel{p}{\rightarrow} \psi)$ increases whenever $\eta$ decreases with respect to set inclusion. 
(ii) If $(X, d)$ is a compact metric space, we can choose the canonical base $\{[d<\varepsilon] ; \varepsilon>0\}$ to obtain the definition given in the introduction.

(iii) If $X$ is a compact Hausdorff space, then $\left\{\bigcup_{U \in \mathcal{U}} U^{2} ; \mathcal{U}\right.$ finite open cover of $\left.X\right\}$ is a base of the uniformity of $X$. Using this base one obtains the definitions used in [55].

(iv) Consider the continuous rotation $\mathbb{R} \times \mathbb{T} \rightarrow \mathbb{T}:(g, x) \mapsto g+x \bmod 1$ with $\mathbb{T}:=\mathbb{R} / \mathbb{Z}$. There holds $\log \left(\operatorname{cov}_{\mathbb{T}}[d<\delta]\right)=\log \left(\operatorname{cov}_{\mathbb{T}}\left[d_{B_{\varepsilon}}<\delta\right]\right)$ for all $\varepsilon, \delta>0$, where $B_{\varepsilon}$ denotes the centred closed ball of radius $\varepsilon$. We thus obtain that $A \mapsto \log \left(\operatorname{cov}_{\mathbb{T}}\left([d<\delta]_{A}\right)\right) \mu(A)^{-1}$ is not bounded, whenever $\delta$ is chosen small enough. This observation is the reason why we can not assume the boundedness of $\mathcal{K}(G) \ni A \mapsto f(A)(\mu(A))^{-1}$ like in [47]. Compare with Remark 3.1.

\subsection{Relative Topological Entropy via Spanning and Separating Sets}

It is well known that one can also define topological entropy of $\mathbb{Z}$-actions on compact metric spaces in terms of separated and of spanning sets $[10,11]$. In [30] this approach is generalized to $\mathbb{Z}$-actions of compact Hausdorff spaces. As this approach is important in the context of aperiodic order $[9,23,34]$ we give a brief recap. Consider a compact Hausdorff space $X$.

Definition 4.7 For $\eta \in \mathbb{U}_{X}$ a subset $S \subseteq X$ is called $\eta$-separated, if for every $s \in S$ there is no further element in $S$ that is $\eta$-close to $s$. Furthermore we say that $S \subseteq X$ is $\eta$-spanning for $M \subseteq X$, if for all $m \in M$ there is $s \in S$ such that $s$ is $\eta$-close to $m$ or $m$ is $\eta$-close to $s$.

Remark 4.8 A subset $S$ of a metric space $(X, d)$ is $[d<\varepsilon]$-separated, if any two distinct points in $S$ are at least $\varepsilon$ apart, i.e. $d(x, y) \geq \varepsilon$ for all $x, y \in S$ with $x \neq y$. Furthermore $S$ is $[d<\varepsilon]$-spanning for $M \subseteq X$, iff for every $m \in M$ there is $s \in S$ such that $d(s, m)<\varepsilon$.

With similar arguments as used in metric spaces we obtain the following lemma.

Lemma 4.9 For $\eta \in \mathbb{U}_{X}$ and $M \subseteq X$ the cardinality of every $\eta$-separated subset $S \subseteq M$ is bounded from above by $\operatorname{cov}_{M}(\eta)<\infty$. In particular there are finite $\eta$-separated subsets of $M$ of maximal cardinality. Every $\eta$-separated subset $S \subseteq M$ of maximal cardinality is $\eta$-spanning for $M$. In particular there are finite subsets of $M$ that are $\eta$-spanning for $M$.

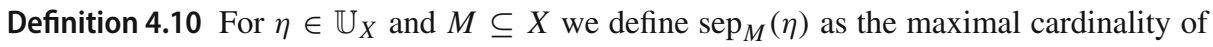
a subset of $M$ that is $\eta$-separated and $\operatorname{spa}_{M}(\eta)$ as the minimal cardinality of a subset of $M$ that is $\eta$-spanning for $M$. For a map $p: X \rightarrow Y$ to some set $Y$ we define

$$
\operatorname{sep}_{p}(\eta):=\sup _{y \in Y} \operatorname{sep}_{p^{-1}(y)}(\eta) \text { and } \operatorname{spa}_{p}(\eta):=\sup _{y \in Y} \operatorname{spa}_{p^{-1}(y)}(\eta) .
$$

Unfortunately the Ornstein-Weiss lemma can not be applied directly to these notions. Nevertheless we use $\operatorname{cov}_{p}$ to show that $\operatorname{spa}_{p}$ and sep $_{p}$ can be used to define entropy independently from the choice of a Van Hove net. A straight forward argument shows the following.

Lemma 4.11 Let $\eta \in \mathbb{U}_{X}$ and $p: X \rightarrow Y$ be a map to a set $Y$. Then there exists an entourage $\vartheta \in \mathbb{U}_{X}$ with $\vartheta \subseteq \eta$ such that for every compact $A \subseteq G$ there holds

$$
\operatorname{cov}_{p}\left(\eta_{A}\right) \leq \operatorname{spa}_{p}\left(\vartheta_{A}\right) \leq \operatorname{sep}_{p}\left(\vartheta_{A}\right) \leq \operatorname{cov}_{p}\left(\vartheta_{A}\right)
$$

Theorem 4.12 Let $\varphi: G \times X \rightarrow X$ be an action of $G$, on a compact Hausdorff space $X$. Let furthermore $\psi$ be a factor of $\varphi$ with factor map $p$. There holds

$$
\mathrm{E}(\varphi \stackrel{p}{\rightarrow} \psi)=\sup _{\eta \in \mathbb{B}_{X}} \limsup _{i \in I} \frac{\log \left(\operatorname{spa}_{p}\left(\eta_{A_{i}}\right)\right)}{\mu\left(A_{i}\right)}=\sup _{\eta \in \mathbb{B}_{X}} \limsup _{i \in I} \frac{\log \left(\operatorname{sep}_{p}\left(\eta_{A_{i}}\right)\right)}{\mu\left(A_{i}\right)}
$$


for any Van Hove net $\left(A_{i}\right)_{i \in I}$ in $G$ and any base $\mathbb{B}_{X}$ of $\mathbb{U}_{X}$. Furthermore the statement remains valid after replacing the limit superior by a limit inferior.

Proof As $\lim \sup _{i \in I} \log \left(\operatorname{spa}_{p}\left(\eta_{A_{i}}\right)\right) / \mu\left(A_{i}\right)$ and the other similar terms are increasing, whenever $\eta$ is decreasing with respect to set inclusion, it suffices to show the statement for $\mathbb{B}_{X}=\mathbb{U}_{X}$. By Lemma 4.11 it is immediate that for any $\eta \in \mathbb{U}_{X}$ there holds

$$
\mathrm{E}(\eta \mid \varphi \stackrel{p}{\rightarrow} \psi) \leq \sup _{\vartheta \in \mathbb{U}_{X}} \limsup _{i \in I} \frac{\log \left(\operatorname{spa}_{p}\left(\vartheta_{A_{i}}\right)\right)}{\mu\left(A_{i}\right)} \leq \mathrm{E}(\varphi \stackrel{p}{\rightarrow} \psi) .
$$

Taking the supremum over $\eta$ yields the first equality. Similar arguments show the statements about sep and the limit inferior.

\section{Relative Topological Entropy via Lattices}

In this section we provide a proof for Theorem 1.4. Recall that for a map $f: A \rightarrow B$ and $M \subseteq A$ we denote by $\left.f\right|_{M}$ the restriction $\left.f\right|_{M}: M \rightarrow B: a \mapsto f(a)$. As before we assume $G$ to satisfy the Ornstein-Weiss lemma.

Proposition 5.1 Let $\Lambda$ be a relatively dense subset of $G$ and let $\left(A_{i}\right)_{i \in I}$ be a Van Hove net. Set $F_{i}:=A_{i} \cap \Lambda$. Let furthermore $f: \mathcal{K}(G) \rightarrow \mathbb{R}$ be a subadditive, right invariant and monotone mapping. If $\Lambda$ has a well defined uniform density dens $(\Lambda)$, then there holds

$$
\lim _{i \in I} \frac{f\left(A_{i}\right)}{\mu\left(A_{i}\right)}=\operatorname{dens}(\Lambda) \lim _{i \in I} \frac{f\left(K F_{i}\right)}{\left|F_{i}\right|} .
$$

Proof Let $K$ be a compact and symmetric subset of $G$ that contains $e_{G}$ and such that $K \Lambda=G$. Let furthermore $M$ be a compact and symmetric neighbourhood of $K$. Then there holds

$$
1 \geq \frac{\mu\left(\overline{A_{i} \backslash \partial_{M} A_{i}}\right)}{\mu\left(A_{i}\right)} \geq \frac{\mu\left(A_{i} \backslash \partial_{M} A_{i}\right)}{\mu\left(A_{i}\right)} \geq \frac{\mu\left(A_{i}\right)-\mu\left(\partial_{M} A_{i}\right)}{\mu\left(A_{i}\right)} \rightarrow 1 .
$$

Thus $\lim _{i \in I} \mu\left(\overline{A_{i} \backslash \partial_{M} A_{i}}\right) / \mu\left(A_{i}\right)=1$. Furthermore a straight forward argument shows $\left(\overline{A_{i} \backslash \partial_{M} A_{i}}\right)^{c} \subseteq\left(A_{i} \backslash \partial_{M} A_{i}\right)^{c}=M \overline{A_{i}^{c}}$. Thus for $C \subseteq G$ compact we obtain

$$
\partial_{C} \overline{A_{i} \backslash \partial_{M} A_{i}}=C \overline{A_{i} \backslash \partial_{M} A_{i}} \cap C \overline{\overline{A_{i} \backslash \partial_{M} A_{i}}} \subseteq C A_{i} \cap C M \overline{A_{i}^{c}} \subseteq \partial_{C M} A_{i}
$$

and we obtain $\left(\overline{A_{i} \backslash \partial_{M} A_{i}}\right)_{i \in I}$ to be a Van Hove net in $G$. As $M$ is a compact neighbourhood we obtain $\overline{A_{i} \backslash \partial_{M} A_{i}} \subseteq A_{i} \backslash \partial_{K} A_{i}$ and one easily shows $A_{i} \backslash \partial_{K} A_{i} \subseteq K F_{i}$. From Proposition 2.3 we know that $\left(K A_{i}\right)_{i \in I}$ is a Van Hove net in $G$ with $\lim _{i \in I} \mu\left(K A_{i}\right) / \mu\left(A_{i}\right)=1$ and compute

$$
\begin{aligned}
\lim _{i \in I} \frac{f\left(A_{i}\right)}{\mu\left(A_{i}\right)} & =\lim _{i \in I} \frac{f\left(\overline{A_{i} \backslash \partial_{M} A_{i}}\right)}{\mu\left(\overline{A_{i} \backslash \partial_{M} A_{i}}\right)} \leq \liminf _{i \in I} \frac{f\left(K F_{i}\right)}{\mu\left(A_{i}\right)} \\
& \leq \limsup _{i \in I} \frac{f\left(K F_{i}\right)}{\mu\left(A_{i}\right)} \leq \lim _{i \in I} \frac{f\left(K A_{i}\right)}{\mu\left(K A_{i}\right)}=\lim _{i \in I} \frac{f\left(A_{i}\right)}{\mu\left(A_{i}\right)} .
\end{aligned}
$$

Thus the statement follows from $\operatorname{dens}(\Lambda)=\lim _{i \in I} \frac{\left|F_{i}\right|}{\mu\left(A_{i}\right)}$.

Theorem 5.2 Let $\varphi$ be an action of $G$ on a compact Hausdorff space X. Let furthermore $\psi$ be a factor of $\varphi$ via factor map $p: X \rightarrow Y$. Let $\Lambda$ be a relatively dense subset of $G$ and let $\left(A_{i}\right)_{i \in I}$ be a Van Hove net. Set $F_{i}:=A_{i} \cap \Lambda$. 
(i) If $\Lambda$ has a well defined uniform density dens $(\Lambda)$, then there holds

$$
\begin{aligned}
\mathrm{E}(\varphi \stackrel{p}{\rightarrow} \psi) & =\operatorname{dens}(\Lambda) \sup _{\eta \in \mathbb{U}_{X}} \liminf _{i \in I} \frac{\log \left(\operatorname{cov}_{p}\left(\eta_{F_{i}}\right)\right)}{\left|F_{i}\right|} \\
& =\operatorname{dens}(\Lambda) \sup _{\eta \in \mathbb{U}_{X}} \limsup \frac{\log \left(\operatorname{cov}_{p}\left(\eta_{F_{i}}\right)\right)}{\left|F_{i}\right|}
\end{aligned}
$$

and these statements remain valid if we consider $\operatorname{sep}_{p}$ and $\mathrm{spa}_{p}$.

(ii) If $\Lambda$ is a uniform lattice, then there holds

$$
\mathrm{E}(\varphi \stackrel{p}{\rightarrow} \psi)=\operatorname{dens}(\Lambda) \mathrm{E}\left(\left.\left.\varphi\right|_{\Lambda \times X} \stackrel{p}{\rightarrow} \psi\right|_{\Lambda \times Y}\right)
$$

Proof The second equality in (i) follows from the first as in the proof of Theorem 4.12. To show the first equality consider a compact subset $K \subseteq G$ with $K \Lambda=G$. From Proposition 5.1 we obtain

$$
\begin{aligned}
\mathrm{E}(\varphi \stackrel{p}{\rightarrow} \psi) & =\sup _{\eta \in \mathbb{U}_{X}} \lim _{i \in I} \frac{\log \left(\operatorname{cov}_{p}\left(\eta_{A_{i}}\right)\right)}{\mu\left(A_{i}\right)} \\
& =\sup _{\eta \in \mathbb{U}_{X}} \lim _{i \in I} \frac{\log \left(\operatorname{cov}_{p}\left(\eta_{K} F_{i}\right)\right)}{\left|F_{i}\right|} \operatorname{dens}(\Lambda) \\
& \leq \sup _{\eta \in \mathbb{U}_{X}} \lim _{i \in I} \frac{\log \left(\operatorname{cov}_{p}\left(\left(\eta_{K}\right) F_{i}\right)\right)}{\left|F_{i}\right|} \operatorname{dens}(\Lambda) \\
& \leq \sup _{\kappa \in \mathbb{U}_{X}} \liminf _{i \in I} \frac{\log \left(\operatorname{cov}_{p}\left(\kappa_{F_{i}}\right)\right)}{\left|F_{i}\right|} \operatorname{dens}(\Lambda) \\
& \leq \sup _{\kappa \in \mathbb{U}_{X}} \lim _{i \in I} \sup \frac{\log \left(\operatorname{cov}_{p}\left(\kappa_{F_{i}}\right)\right)}{\left|F_{i}\right|} \operatorname{dens}(\Lambda) \\
& \leq \sup _{\kappa \in \mathbb{U}_{X}} \lim _{i \in I} \frac{\log \left(\operatorname{cov}_{p}\left(\kappa_{A_{i}}\right)\right)}{\mu\left(A_{i}\right)} .
\end{aligned}
$$

This shows (i). To show (ii) note first that every uniform lattice has a well defined uniform density which is given by $\operatorname{dens}(\Lambda)=\mu(C)^{-1}$ for any fundamental domain $C \subseteq G$ of $\Lambda$. We use Theorem 3.3 to obtain that for any Van Hove net $\left(E_{i}\right)_{i \in I}$ in $\Lambda$ there holds $\mu(C) \mathrm{E}(\varphi \stackrel{p}{\rightarrow} \psi)=\sup _{\eta \in \mathbb{U}_{X}} \lim _{i \in I} \log \left(\operatorname{cov}_{p}\left(\eta_{\bar{C} E_{i}}\right)\right)\left|E_{i}\right|^{-1}$. As

$$
\mathrm{E}\left(\left.\left.\varphi\right|_{\Lambda \times X} \stackrel{p}{\rightarrow} \psi\right|_{\Lambda \times Y}\right)=\sup _{\eta \in \mathbb{U}_{X}} \lim _{i \in I} \frac{\log \left(\operatorname{cov}_{p}\left(\eta_{\bar{C} E_{i}}\right)\right)}{\left|E_{i}\right|}
$$

follows similarly as in (i) we obtain the statement.

Remark 5.3 Note that for $n \in \mathbb{N}$ the set $\{0, \ldots, n-1\}$ is a fundamental domain for the uniform lattice $n \mathbb{Z}$ in $\mathbb{Z}$. We thus obtain from Theorem 5.2 for every homeomorphism $f: X \rightarrow X$ the well known formula $n \mathrm{E}(f)=\mathrm{E}\left(f^{n}\right)$. Here $\mathrm{E}(g)$ abbreviates the topological entropy of the flow $\varphi: \mathbb{Z} \times X \rightarrow X$ with $\varphi(n, x)=g^{n}(x)$ for a homeomorphism $g: X \rightarrow X$. Thus $\mathrm{E}\left(f^{n}\right)$ is the entropy of the flow $(m, x) \mapsto f^{m}(x)$ restricted to $n \mathbb{Z} \times X \rightarrow X$.

\section{Bowen's Formula}

In this section we present a proof of Theorem 1.5. The following consequences can be drawn directly from this theorem and the literature [58]. 
Remark 6.1 If we take $\rho$ as the action on a one point space, we obtain the classical formulation of Bowen's formula for the entropy of factors from the second inequality. Furthermore we obtain $\mathrm{E}(\varphi)=\mathrm{E}(\varphi \stackrel{p}{\rightarrow} \psi)$, whenever $\mathrm{E}(\psi)=0$; and $\mathrm{E}(\varphi)=\mathrm{E}(\psi)$, whenever $\mathrm{E}(\varphi \stackrel{p}{\rightarrow} \psi)=$ 0 . If we assume $G$ to be non-compact and $X$ and $Y$ to be compact metrizable spaces the latter assumption $\mathrm{E}(\varphi \stackrel{p}{\rightarrow} \psi)=0$ is satisfied under each of the following conditions.

(i) $p$ is a distal factor map, i.e. for $y \in Y$ all pairs of distinct points in $p^{-1}(y)$ are distal. ${ }^{10}$

(ii) $p$ is a countable to one factor map, i.e. for $y \in Y$ the fiber $p^{-1}(y)$ is countable.

Proof The statement in (i) implies $p$ to be a distal factor map from the action $\left.\varphi\right|_{\Lambda \times X}$ to $\left.\psi\right|_{\Lambda \times Y}$ for every countable uniform lattice $\Lambda \subseteq G$. We thus obtain the statement from [58, Corollary 6.7] and Theorem 5.2. We get (ii) directly from [58, Theorem 5.7] and Theorem 5.2. Note that we restrict to metric spaces, as the statements in [58] are presented for compact metric spaces.

\subsection{Measure Theoretic Relative Entropy for Actions of Countable Discrete Amenable Groups}

We give a brief introduction into the theory of measure theoretical relative entropy of actions of discrete amenable groups, presented in various texts, such as [14,21,41,56-58], in order to state the variational principle and the Rohlin-Abramov theorem. Let $X$ be a compact Hausdorff space. By $\mathcal{B}_{X}$ we denote the Borel $\sigma$-algebra. Furthermore we denote the set of all regular Borel probability measures by $\mathcal{M}(X)$. By the Riesz-Markov theorem we can identify $\mathcal{M}(X)$ with the convex cone base of all positive functionals in $C(X)$ that map the unit $(X \rightarrow \mathbb{R} ; x \mapsto 1)$ to 1 . We equip $\mathcal{M}(X)$ with the restricted weak*-topology and obtain a compact topological space from the Banach-Alaoglu theorem. For a reference see [22, Theorem E.11].

A family $\alpha$ of pairwise disjoint Borel-measurable non empty subsets of $X$ with union $X$ is called a measurable partition of $X$. We denote the set of all finite partitions of $X$ by $\mathcal{P}_{X}$. The refinement of two partitions $\alpha, \beta \in \mathcal{P}_{X}$ is the partition $\alpha \vee \beta:=\{A \cap B ; A \in$ $\alpha$ and $B \in \beta\} \backslash\{\emptyset\}$. Similarly the refinement of a finite number of partitions is defined. Let $\varphi: \Lambda \times X \rightarrow X$ be an action of a countable discrete amenable group $\Lambda$ on a compact Hausdorff space. For a finite subset $F \subseteq \Lambda$ we denote by $\alpha_{F}$ the refinement of the partitions $\left\{\left(\varphi^{g}\right)^{-1}(A) ; A \in \alpha\right\}$, where $g$ ranges over $F$. A measure $v \in \mathcal{M}(X)$ is called $\varphi$ - invariant, if $v(A)=v\left(\varphi^{g}(A)\right)$ for every $g \in G$. We denote by $\mathcal{M}_{\varphi}$ the set of all $\varphi$-invariant $v \in \mathcal{M}(X)$. Every continuous map $p: X \rightarrow Y$ to some compact Hausdorff space is measurable with respect to the Borel $\sigma$-algebras and $p^{-1}\left(\mathcal{B}_{Y}\right)$ is a sub- $\sigma$-algebra of $\mathcal{B}_{X}$. For $A \in \mathcal{B}_{X}$ and $v \in \mathcal{M}_{\varphi}$ let $\mathbb{E}_{v, p}\left(\chi_{A}\right)$ be the conditional expectation of the characteristic function $\chi_{A}$ of $A$ with respect to $p^{-1}\left(\mathcal{B}_{X}\right)$. For $\alpha \in \mathcal{P}_{X}$ we define

$$
H_{\nu, p}(\alpha):=-\sum_{A \in \alpha} \int_{X} \mathbb{E}_{\nu, p}\left(\chi_{A}\right) \log \left(\mathbb{E}_{\nu, p}\left(\chi_{A}\right)\right) d \nu .
$$

As presented in [58] the Ornstein-Weiss lemma can be applied to $\mathcal{F}(\Lambda) \ni F \mapsto H_{v, p}\left(\alpha_{F}\right)$ for every $\alpha \in \mathcal{P}_{X}$ to obtain that $\mathrm{E}_{v}(\alpha \mid \varphi \stackrel{p}{\rightarrow} \psi):=\lim _{i \in I} H_{v, p}\left(\alpha_{F_{i}}\right)\left|F_{i}\right|^{-1}$ exists and is independent of the choice of the Van Hove net $\left(F_{i}\right)_{i \in I}$ in $\Lambda$. The relative measure theoretical entropy of $\varphi$ under the condition $\psi$ is given by

10 We call two points $x, x^{\prime} \in X$ distal, whenever there is $\eta \in \mathbb{U}_{X}$ such that $\left(x, x^{\prime}\right) \notin \eta_{g}$ for all $g \in G$. 


$$
\mathrm{E}_{v}(\varphi \stackrel{p}{\rightarrow} \psi):=\sup _{\alpha \in \mathcal{P}_{X}} \mathrm{E}_{v}(\alpha \mid \varphi \stackrel{p}{\rightarrow} \psi)
$$

The Rohlin-Abramov theorem for actions of countable amenable groups appeared firstly in [57] and can be also found in [14,20,25,58]. For a continuous surjective mapping $p: X \rightarrow Y$ between compact Hausdorff spaces and a $v \in \mathcal{M}(X)$ the push forward measure is defined by $p_{*} v \in \mathcal{M}(Y)$ by $p_{*} v(M):=v\left(p^{-1}(M)\right)$ for $M \in \mathcal{B}_{Y}$.

Proposition 6.2 (Rohlin-Abramov Theorem) Let $\varphi, \psi$ and $\rho$ be actions of a countable discrete amenable group $\Lambda$ on compact Hausdorff spaces $X, Y$ and $Z$ respectively. Let $\psi$ be a factor of $\varphi$ via the factor map $p$ and $\rho$ be a factor of $\psi$ via the factor map $q$, i.e. $\varphi \stackrel{p}{\rightarrow} \psi \stackrel{q}{\rightarrow} \rho$. Then for $\left(v_{X}, v_{Y}, v_{Z}\right) \in \mathcal{M}_{\varphi}(X) \times \mathcal{M}_{\psi}(Y) \times \mathcal{M}_{\rho}(Z)$ that satisfy $p_{*} v_{X}=v_{Y}$ and $q_{*} v_{Y}=v_{Z}$ there holds

$$
\mathrm{E}_{v_{X}}(\varphi \stackrel{q \circ p}{\rightarrow} \rho)=\mathrm{E}_{v_{X}}(\varphi \stackrel{p}{\rightarrow} \psi)+\mathrm{E}_{v_{Y}}(\psi \stackrel{q}{\rightarrow} \rho) .
$$

The following version of a variational principle is valid in our context.

Theorem 6.3 Let $\varphi$ be an action of an amenable group $G$, containing a countable uniform lattice $\Lambda$, on a compact Hausdorff space $X$ and let $\psi$ be a factor of $\varphi$ via $p: X \rightarrow Y$. Then

$$
\mathrm{E}(\varphi \stackrel{p}{\rightarrow} \psi)=\mu(C) \sup _{\nu \in \mathcal{M}_{\left.\varphi\right|_{\Lambda \times X}}} \mathrm{E}_{\nu}\left(\left.\left.\varphi\right|_{\Lambda \times X} \stackrel{p}{\rightarrow} \psi\right|_{\Lambda \times Y}\right) .
$$

Proof By Theorem 5.2 it remains to show that

$$
\mathrm{E}\left(\left.\left.\varphi\right|_{\Lambda \times X} \stackrel{p}{\rightarrow} \psi\right|_{\Lambda \times Y}\right)=\sup _{\nu \in \mathcal{M}_{\left.\varphi\right|_{\Lambda \times X}}} \mathrm{E}_{\nu}\left(\left.\left.\varphi\right|_{\Lambda \times X} \stackrel{p}{\rightarrow} \psi\right|_{\Lambda \times Y}\right) .
$$

A proof can be found in [42,53] and goes back to [17,26,27].

For the proof of Theorem 1.5 we need a further ingredient. By a standard KrylovBogolyubov procedure one obtains the following.

Lemma 6.4 Let $\varphi$ be an action of a discrete amenable group $\Lambda$ on a compact topological space $X$ and $\psi$ be a factor of $\varphi$ via factor map $p$. Then the restricted push forward operation $p_{*}: \mathcal{M}_{\varphi} \rightarrow \mathcal{M}_{\psi}$ is surjective.

\subsection{Proof of Theorem 1.5}

Proof of Theorem 1.5 Let $\varphi, \psi$ and $\rho$ be actions of an amenable group containing a countable uniform lattice $\Lambda$ on compact Hausdorff spaces $X, Y$ and $Z$ respectively. Assume $\varphi \stackrel{p}{\rightarrow} \psi \stackrel{q}{\rightarrow}$ $\rho$. Let $C$ be a fundamental domain of $\Lambda$. We abbreviate $\varphi^{\Lambda}:=\left.\varphi\right|_{\Lambda \times X}, \psi^{\Lambda}$ and $\rho^{\Lambda}$ for the restrictions of the actions to the lattice. From Theorem 6.3 and Proposition 6.2 we obtain

$$
\begin{aligned}
\mathrm{E}(\varphi \stackrel{p}{\rightarrow} \psi) & =\mu(C) \sup _{v \in \mathcal{M}_{\varphi^{\Lambda}}} \mathrm{E}_{\nu}\left(\varphi^{\Lambda} \stackrel{p}{\rightarrow} \psi^{\Lambda}\right) \\
& \leq \mu(C) \sup _{\nu \in \mathcal{M}_{\varphi^{\Lambda}}}\left(\mathrm{E}_{\nu}\left(\varphi^{\Lambda} \stackrel{p}{\rightarrow} \psi^{\Lambda}\right)+\mathrm{E}_{p_{*} v}\left(\psi^{\Lambda} \stackrel{q}{\rightarrow} \rho^{\Lambda}\right)\right) \\
& =\mu(C) \sup _{\nu \in \mathcal{M}_{\varphi^{\Lambda}}} \mathrm{E}_{\nu}\left(\varphi^{\Lambda} \stackrel{q \circ p}{\rightarrow} \psi^{\Lambda}\right)=\mathrm{E}(\varphi \stackrel{q \circ p}{\rightarrow} \rho) .
\end{aligned}
$$


By Lemma 6.4 we observe

$$
\mathrm{E}\left(\psi^{\Lambda} \stackrel{q}{\rightarrow} \rho^{\Lambda}\right)=\mu(C) \sup _{\omega \in \mathcal{M}_{\psi} \Lambda} \mathrm{E}_{\omega}\left(\psi^{\Lambda} \stackrel{q}{\rightarrow} \rho^{\Lambda}\right)=\mu(C) \sup _{\nu \in \mathcal{M}_{\varphi} \Lambda}\left(\mathrm{E}_{p_{*} \nu}\left(\psi^{\Lambda} \stackrel{q}{\rightarrow} \rho^{\Lambda}\right)\right)
$$

and argue similarly to obtain $\mathrm{E}(\psi \stackrel{q}{\rightarrow} \rho) \leq \mathrm{E}(\varphi \stackrel{q \circ p}{\rightarrow} \rho)$. To finish the proof we compute

$$
\begin{aligned}
\mathrm{E}(\varphi \stackrel{q \circ p}{\rightarrow} \rho) & =\mu(C) \sup _{v \in \mathcal{M}_{\varphi^{\Lambda}}} \mathrm{E}_{v}\left(\varphi^{\Lambda} \stackrel{q \circ p}{\rightarrow} \psi^{\Lambda}\right) \\
& =\mu(C) \sup _{v \in \mathcal{M}_{\varphi^{\Lambda}}}\left(\mathrm{E}_{v}\left(\varphi^{\Lambda} \stackrel{p}{\rightarrow} \psi^{\Lambda}\right)+\mathrm{E}_{p_{*} v}\left(\psi^{\Lambda} \stackrel{q}{\rightarrow} \rho^{\Lambda}\right)\right) \\
& \leq \mu(C) \sup _{\nu \in \mathcal{M}_{\varphi^{\Lambda}}}\left(\mathrm{E}_{v}\left(\varphi^{\Lambda} \stackrel{p}{\rightarrow} \psi^{\Lambda}\right)\right)+\mu(C) \sup _{\omega \in \mathcal{M}_{\psi^{\Lambda}}}\left(\mathrm{E}_{\omega}\left(\psi^{\Lambda} \stackrel{q}{\rightarrow} \rho^{\Lambda}\right)\right) \\
& =\mathrm{E}(\varphi \stackrel{p}{\rightarrow} \psi)+\mathrm{E}(\psi \stackrel{q}{\rightarrow} \rho) .
\end{aligned}
$$

Acknowledgements Open Access funding provided by Projekt DEAL. The author would like to thank Yves de Cornulier for pointing out Example 3.4 to him and is grateful for lively discussions with Felix H. Pogorzelski on the topic of approximate lattices. Furthermore the author would like to thank Tobias Jäger for his patient supervision and several useful hints.

Open Access This article is licensed under a Creative Commons Attribution 4.0 International License, which permits use, sharing, adaptation, distribution and reproduction in any medium or format, as long as you give appropriate credit to the original author(s) and the source, provide a link to the Creative Commons licence, and indicate if changes were made. The images or other third party material in this article are included in the article's Creative Commons licence, unless indicated otherwise in a credit line to the material. If material is not included in the article's Creative Commons licence and your intended use is not permitted by statutory regulation or exceeds the permitted use, you will need to obtain permission directly from the copyright holder. To view a copy of this licence, visit http://creativecommons.org/licenses/by/4.0/.

\section{References}

1. Axel, F., Gratias, D.: Beyond quasicrystals. (1995)

2. Baake, M.: Directions in Mathematical Quasicrystals, vol. 13. American Mathematical Soc., Providence (2000)

3. Bake, M., Grimm, U.: Aperiodic Order, vol. 1. Cambridge University Press, Cambridge (2013)

4. Baake, M., Huck, C.: Ergodic properties of visible lattice points. Proc. Steklov Inst. Math. 288(1), 165-188 (2015)

5. Björklund, M., Hartnick, T., et al.: Approximate lattices. Duke Math. J. 167(15), 2903-2964 (2018)

6. Björklund, M., Hartnick, T., Pogorzelski, F.: Aperiodic order and spherical diffraction, i: auto-correlation of regular model sets. Proc. Lond. Math. Soc. 116(4), 957-996 (2018)

7. Baake, M., Lenz, D.: Dynamical systems on translation bounded measures: pure point dynamical and diffraction spectra. Ergodic Theory Dyn. Syst. 24(6), 1867-1893 (2004)

8. Bake, M., Lenz, D.: Spectral notions of aperiodic order. Discret. Contin. Dyn. Syst. S 10(2), 161-190 (2017)

9. Baake, M., Lenz, D., Richard, C.: Pure point diffraction implies zero entropy for delone sets with uniform cluster frequencies. Lett. Math. Phys. 82(1), 61-77 (2007)

10. Bowen, R.: Entropy for group endomorphisms and homogeneous spaces. Trans. Am. Math. Soc. 153, 401-414 (1971)

11. Brin, M., Stuck, G.: Introduction to Dynamical Systems. Cambridge University Press, Cambridge (2002)

12. Cornulier, Y., de La Harpe, P.: Metric geometry of locally compact groups. In: Winner of the 2016 EMS Monograph Award. EMS Tracts in Mathematics, 25. European Mathematical Society (EMS), Zürich, 2016. pp. viii+235, ISBN: 978-3-03719-166-8 
13. Ceccherini-Silberstein, T., Coornaert, M., Krieger, F.: An analogue of Fekete's lemma for subadditive functions on cancellative amenable semigroups. Journal d'analyse mathématique 124(1), 59-81 (2014)

14. Danilenko, A.I.: Entropy theory from the orbital point of view. Monatshefte für Mathematik 134(2), 121-141 (2001)

15. Deitmar, A., Echterhoff, S.: Principles of Harmonic Analysis. Springer, New York (2014)

16. Downarowicz, T., Huczek, D., Zhang, G.: Tilings of amenable groups. Journal für die Reine und Angewandte Mathematik (Crelles Journal) 2019(747), 277-298 (2019)

17. Dinaburg, E.I.: A connection between various entropy characterizations of dynamical systems. Izv. Akad. Nauk SSSR Ser. Mat 35(324-366), 13 (1971)

18. Dunford, N., Schwartz, J.T.: Linear Operators Part I: General Theory, vol. 7. Interscience publishers, New York (1958)

19. Dikranjan, D., Sanchis, M., Virili, S.: New and old facts about entropy in uniform spaces and topological groups. Topol. Appl. 159(7), 1916-1942 (2012)

20. Dooley, A., Zhang, G.: Local entropy theory of a random dynamical system, vol. 233. American Mathematical Society, Providence (2015)

21. Downarowicz, T., Zhang, G.: Symbolic extensions of amenable group actions and the comparison property. arXiv preprint arXiv:1901.01457, (2019). Accepted for publication in Memoirs of the American Mathematical Society

22. Eisner, T., Farkas, B., Haase, M., Nagel, R.: Operator Theoretic Aspects of Ergodic Theory, vol. 272. Springer, New York (2015)

23. Fuhrmann, G., Glasner, E., Jäger, T., Oertel, C.: Irregular model sets and tame dynamics. arXiv preprint arXiv:1811.06283, (2018)

24. Folland, G.B.: Real Analysis: Modern Techniques and Their Applications. Wiley, Hoboken (2013)

25. Glasner, E., Thouvenot, J.-P., Weiss, B.: Entropy theory without a past. Ergodic Theory Dyn. Syst. 20(5), 1355-1370 (2000)

26. Goodwyn, L.W.: Topological entropy bounds measure-theoretic entropy. Proc. Am. Math. Soc. 23(3), 679-688 (1969)

27. Goodman, T.N.T.: Relating topological entropy and measure entropy. Bull. Lond. Math. Soc. 3(2), 176180 (1971)

28. Gouvêa, F.Q.: p-adic numbers. In: p-adic Numbers, pp. 43-85. Springer, New York, (1997)

29. Gromov, M.: Topological invariants of dynamical systems and spaces of holomorphic maps: I. Math. Phys. Anal. Geom. 2(4), 323-415 (1999)

30. Hood, B.M.: Topological entropy and uniform spaces. J. Lond. Math. Soc. 2(4), 633-641 (1974)

31. Hewitt, E., Ross, K.A.: Abstract Harmonic Analysis: Volume I Structure of Topological Groups Integration Theory Group Representations, vol. 115. Springer, New York (2012)

32. Huck, C., Richard, C.: On pattern entropy of weak model sets. Discrete Comput. Geom. 54(3), 741-757 (2015)

33. Huang, W., Ye, X., Zhang, G.: Local entropy theory for a countable discrete amenable group action. J. Funct. Anal. 261(4), 1028-1082 (2011)

34. Jäger, T., Lenz, D., Oertel, C.: Model sets with positive entropy in Euclidean cut and project schemes. Ann. Sci. Norm. Sup. 52(4e), 1073-1106 (2019)

35. Kelley, J.L.: General Topology. Courier Dover Publications, New York (2017)

36. Krieger, F.: The Ornstein-Weiss lemma for discrete amenable groups. Max Planck Inst. Math. Bonn. 48, 2010 (2010)

37. Lindenstrauss, E., Weiss, B.: Mean topological dimension. Isr. J. Math. 115(1), 1-24 (2000)

38. Machado, S.: Approximate lattices and meyer sets in nilpotent lie groups. arXiv preprint arXiv: $1810.10870,(2018)$

39. Meyer, Y.: Algebraic Numbers and Harmonic Analysis, vol. 2. Elsevier, Amsterdam (1972)

40. Munkres, J.R.: Topology. Prentice Hall, New York (2000)

41. Ollagnier, J.M.: Ergodic Theory and Statistical Mechanics, vol. 1115. Springer, New York (2007)

42. Ollagnier, J.M., Pinchon, D.: The variational principle. Stud. Math. 72, 151-159 (1982)

43. Ornstein, D.S., Weiss, B.: Entropy and isomorphism theorems for actions of amenable groups. Journal d'Analyse Mathématique 48(1), 1-141 (1987)

44. Patera, J.: Quasicrystals and Discrete Geometry, vol. 10. American Mathematical Soc, Providence (1998)

45. Paterson, A.L.T.: Amenability, vol. 29. Providence, American Mathematical Soc. (2000)

46. Pier, J.-P.: Amenable Locally Compact Groups. Wiley, Hoboken (1984)

47. Pogorzelski, F., Schwarzenberger, F.: A banach space-valued ergodic theorem for amenable groups and applications. Journal d'Analyse Mathématique 130(1), 19-69 (2016)

48. Runde, V.: Lectures on Amenability. Springer, New York (2004) 
49. Shechtman, D., Blech, I., Gratias, D., Cahn, J.W.: Metallic phase with long-range orientational order and no translational symmetry. Phys. Rev. Lett. 53(20), 1951 (1984)

50. Schlottmann, M.: Generalized Model Sets and Dynamical Systems. CRM Monograph Series. Citeseer, Princeton (1999)

51. Schneider, F.M.: Topological entropy of continuous actions of compactly generated groups. arXiv preprint arXiv:1502.03980, (2015)

52. Strungaru, N.: Almost periodic measures and meyer sets. Discrete Comput. Geom. 33(3), 483-505 (2005)

53. Stepin, A.M., Tagi-Zade, A.T.: Variational characterization of topological pressure of the amenable groups of transformations. In: Doklady Akademii Nauk, Vol. 254, pp. 545-549. Russian Academy of Sciences, Moscow, (1980)

54. Tempelman, A.: Ergodic Theorems for Group Actions: Informational and Thermodynamical Aspects. Springer, New York (2013)

55. Tagi-Zade, A.T.: Variational characterization of topological entropy of continuous transformation groups. case of actions of $\mathbb{R}^{n}$. Math. Notes Acad. Sci. USSR 49(3), 305-311 (1991)

56. Weiss, B.: Actions of amenable groups. Top. Dyn. Ergod. Theory 310, 226-262 (2003)

57. Ward, T., Zhang, Q.: The Abramov-Rokhlin entropy addition formula for amenable group actions. Monatshefte für Mathematik. 114(3-4), 317-329 (1992)

58. Yan, K.: Conditional entropy and fiber entropy for amenable group actions. J. Differ. Equ. 259(7), 30043031 (2015)

59. Yan, K., Zeng, F.: Topological entropy, pseudo-orbits and uniform spaces. Topol. Appl. 210, 168-182 (2016)

60. Zheng, D., Chen, E.: Bowen entropy for actions of amenable groups. Isr. J. Math. 212(2), 895-911 (2016)

61. Zhou, Y.: Tail variational principle for a countable discrete amenable group action. J. Math. Anal. Appl. 433(2), 1513-1530 (2016)

Publisher's Note Springer Nature remains neutral with regard to jurisdictional claims in published maps and institutional affiliations. 